\title{
HERSCHEL KEY PROGRAM, “DUST, ICE, AND GAS IN TIME” (DIGIT): THE ORIGIN OF MOLECULAR AND ATOMIC EMISSION IN LOW-MASS PROTOSTARS IN TAURUS
}

\author{
Jeong-Eun LeE ${ }^{1,2}$, Jinhee LeE ${ }^{3}$, Seokho LeE ${ }^{1}$, Neal J. Evans II $^{2}$, And Joel D. Green ${ }^{2}$ \\ ${ }^{1}$ Department of Astronomy and Space Science, Kyung Hee University, Yongin-shi, Kyungki-do 449-701, Korea; jeongeun.lee@ khu.ac.kr \\ ${ }^{2}$ Department of Astronomy, University of Texas at Austin, 2515 Speedway, Stop C1400, Austin, TX 78712-1205, USA \\ ${ }^{3}$ Department of Physics and Astronomy, The University of Georgia, Athens, GA 30602-2451, USA \\ Received 2014 April 12; accepted 2014 July 10; published 2014 September 18
}

\begin{abstract}
Six low-mass embedded sources (L1489, L1551-IRS5, TMR1, TMC1-A, L1527, and TMC1) in Taurus have been observed with Herschel-PACS to cover the full spectrum from 50 to $210 \mu \mathrm{m}$ as part of the Herschel key program, "Dust, Ice, and Gas In Time." The relatively low intensity of the interstellar radiation field surrounding Taurus minimizes contamination of the $[\mathrm{C} \mathrm{II}]$ emission associated with the sources by diffuse emission from the cloud surface, allowing study of the [C II] emission from the source. In several sources, the [C $\mathrm{CII}_{\mathrm{II}}$ emission is distributed along the outflow, as is the [O I] emission. The atomic line luminosities correlate well with each other, as do the molecular lines, but the atomic and molecular lines correlate poorly. The relative contribution of $\mathrm{CO}$ to the total gas cooling is constant at $\sim 30 \%$, while the cooling fraction by $\mathrm{H}_{2} \mathrm{O}$ varies from source to source, suggesting different shock properties resulting in different photodissociation levels of $\mathrm{H}_{2} \mathrm{O}$. The gas with a power-law temperature distribution with a moderately high density can reproduce the observed CO fluxes, indicative of CO close to LTE. However, $\mathrm{H}_{2} \mathrm{O}$ is mostly subthermally excited. L1551-IRS5 is the most luminous source $\left(€_{\mathrm{bol}}=24.5 L_{\odot}\right)$ and the [O I] $63.1 \mu \mathrm{m}$ line accounts for more than $70 \%$ of its FIR line luminosity, suggesting complete photodissociation of $\mathrm{H}_{2} \mathrm{O}$ by a J shock. In L1551-IRS5, the central velocity shifts of the [O I] line, which exceed the wavelength calibration uncertainty $\left(\sim 70 \mathrm{~km} \mathrm{~s}^{-1}\right)$ of PACS, are consistent with the known redshifted and blueshifted outflow direction.
\end{abstract}

Key words: astrochemistry - ISM: jets and outflows - molecular processes - stars: protostars techniques: spectroscopic

Online-only material: color figures, figure sets

\section{INTRODUCTION}

Jets and outflows are the most spectacular features of the star formation process. Although infall and accretion are the key processes in star formation, they are difficult to observe directly. Because models predict that jets and outflows correlate with accretion (Shu et al. 1987; Bontemps et al. 1996), they provide indirect probes of accretion. For example, the average outflow force is stronger in Class 0 objects than Class I sources, indicating that the mass accretion rate decreases as the central protostar evolves (Bontemps et al. 1996; Curtis et al. 2010). Analysis of outflows can also probe the energetics and chemistry that occur when the jets carve out the envelope to construct bipolar cavities. Both high-energy photons and shocks can heat the cavity walls farther from the central protostar than would be possible without the cavities (Visser et al. 2012). This bipolar cavity structure has been traced by the scattered emission at short wavelengths (e.g., Tobin et al. 2008) and by maps of outflows with high resolution (e.g., Wu et al. 2009 at $1.3 \mathrm{~mm}$ ).

Recent observations with instruments aboard the Herschel Space Observatory (Pilbratt et al. 2010) strongly suggest that the far-infrared (FIR) line emission is predominantly produced by shocks associated with jets and outflows (Manoj et al. 2013; Karska et al. 2013) although energetic photons produced by the accretion process also contribute to exciting the FIR line (especially CO line) emission (e.g., Visser et al. 2012). In addition, according to the detailed models of Kristensen et al. (2011), Goicoechea et al. (2012), Karska et al. (2013), and Lee et al. (2013), molecules with temperatures of $\sim 100-1000 \mathrm{~K}$ emit most of their luminosity $(\sim 80 \%)$ in the wavelength range of $50-200 \mu \mathrm{m}$, which is covered by the Photodetector Array Camera and Spectrometer (PACS; Poglitsch et al. 2010).

Therefore, covering the spectral energy distribution (SED) from 50 to $210 \mu \mathrm{m}$, the PACS full SED range mode observations of a large number of embedded sources can provide an excellent opportunity to study the dependence of the gas cooling budget on evolutionary phase. However, kinematic constraints cannot usually be obtained from unresolved PACS lines $\left(\delta v \sim 50-100 \mathrm{~km} \mathrm{~s}^{-1}\right)$. Complementary kinematic information

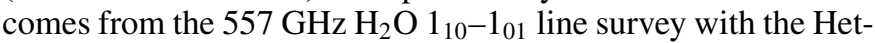
erodyne Instrument for the Far-Infrared on Herschel (HIFI; de Graauw et al. 2010) obtained by the Herschel key programs, "Water in star forming regions with Herschel" (WISH) and "Dust, Ice, and Gas In Time" (DIGIT) carried out toward the same sources that were observed with PACS (Kristensen et al. 2012; Green et al. 2013). According to Kristensen et al. (2012), the $\mathrm{H}_{2} \mathrm{O}$ line emission is correlated with other evolutionary indicators such as $L_{\mathrm{bol}}, T_{\mathrm{bol}}$, and envelope mass as well as the indicators of outflow strength such as outflow momentum flux calculated from the $\operatorname{CO} J=3-2$ line and the line width of the $557 \mathrm{GHz}$ water line. While HIFI can resolve the $557 \mathrm{GHz}$ water line into kinematic components in the protostellar system, the PACS spectra are well suited to study the cooling of the heated gas because all of dominant cooling lines are covered by the observations.

We present PACS spectra toward six embedded young stellar objects (YSOs) in Taurus, from the DIGIT program (PI: N. Evans; Green et al. 2013). Because they are forming in the same environment, that variable can be removed. In particular, the Taurus cloud exists in a region of low excitation by the 
Table 1

Source Information

\begin{tabular}{lccrr}
\hline \hline Name & R.A. & Decl. & $\begin{array}{c}T_{\text {bol }^{\mathrm{a}}} \\
(\mathrm{K})\end{array}$ & $\begin{array}{r}L_{\text {bol }}{ }^{\mathrm{a}} \\
\left(L_{\odot}\right)\end{array}$ \\
\hline L1489 & $04: 04: 42.9$ & $+26: 18: 56.3$ & 226 & 3.5 \\
L1551-IRS5 & $04: 31: 34.1$ & $+18: 08: 04.9$ & 106 & 24.5 \\
TMR1 & $04: 39: 13.9$ & $+25: 53: 20.6$ & 140 & 2.6 \\
TMC1-A & $04: 39: 35.0$ & $+25: 41: 45.5$ & 164 & 2.5 \\
L1527 & $04: 39: 53.9$ & $+26: 03: 09.8$ & 67 & 1.7 \\
TMC1 & $04: 41: 12.7$ & $+25: 46: 35.9$ & 171 & 0.66 \\
\hline
\end{tabular}

Note. ${ }^{a}$ Values from Green et al. (2013).

interstellar radiation field (ISRF), minimizing external radiation effects. These effects can influence the emission, as seen in sources in the Serpens (Dionatos et al. 2013), Corona Aus (Lindberg et al. 2014), and Ophiuchus (H. Je et al., in preparation) clouds. Although all sources in our sample except L1527 are Class I sources, they span a substantial range of $L_{\mathrm{bol}}$ $\left(0.7-24.5 L_{\odot}\right)$ and $T_{\text {bol }}(67-226 \mathrm{~K})$, as noted in Table 1 .

The PACS observations, data reduction, and flux measurement are explained in Section 2. Spatial distributions of lines and their luminosities are described in Section 3, and the analyses of FIR line fluxes with rotation diagrams and the non-LTE LVG code, RADEX (van der Tak et al. 2007) are presented in Section 4. L1551-IRS5, which is the most luminous and peculiar source among our samples, is separately discussed in Section 5, and we discuss the mechanisms for the FIR line emission in Section 6. Finally, the summary of this work will be given in Section 7.

\section{OBSERVATIONS AND FLUX MEASUREMENT}

PACS provides a $5 \times 5$ array of 9..4 × 9.4 spatial pixels (hereafter "spaxels") covering the spectral range from 50 to $210 \mu \mathrm{m}$ with $\lambda / \Delta \lambda \sim 1000-2500$, divided into four segments, covering $\lambda \sim 50-75,70-105,100-145$, and 140-210 $\mu \mathrm{m}$. The PACS spatial resolution ranges from $\sim 9^{\prime \prime}$ at the shortest wavelengths $(50 \mu \mathrm{m})$ to $\sim 18^{\prime \prime}$ at the longest $210 \mu \mathrm{m}$ (refer to PACS observer's manual $2010^{4}$ ).

Information on the Taurus sources covered by the DIGIT program are listed in Table 1. More detailed information on the PACS observations of our targets can be found in Green et al. (2013): basic parameters of protostars $\left(L_{\mathrm{bol}}\right.$ and $\left.T_{\mathrm{bol}}\right)$, the rotation diagrams for the total numbers of each molecule and its rotation temperature, and the correlations between various physical parameters and line properties. The PACS SEDs of these sources are presented in Green et al. (2013), so we present only the continuum subtracted line spectra in Figure 1. The basic data reduction procedures are explained in detail in Green et al. (2013). Among our sources, TMR1, TMC1-A, and L1527 were mispointed (due to a known early mission issue). TMC1-A is mispointed by one spaxel to the east, but the emission is well confined in this single off-center spaxel. TMR1 and L1527 both are shifted to the northeast from the central spaxel; the strongest continuum emission for L1527 is still at the central spaxel while the emission peak is shifted to the NE by one spaxel for TMR1. In TMR1 and L1527, the continuum emission distributes over a few spaxels due to the mispointing. Figure 2 shows the PACS images at the $68 \mu \mathrm{m}$ continuum for the mispointed sources.

\footnotetext{
4 http://herschel.esac.esa.int/Docs/PACS/pdf/pacs_om.pdf
}

Table 2

Correction Factors

\begin{tabular}{llccccc}
\hline \hline & L1489 & L1551-IRS5 & TMR1 & TMC1-A & L1527 & TMC1 \\
\hline Blue $(<100 \mu \mathrm{m})$ & 1.0 & $0.891^{\mathrm{a}}, 0.979^{\mathrm{b}}$ & 1.076 & 1.054 & 1.028 & 0.960 \\
Red $(\geqslant 100 \mu \mathrm{m})$ & 0.939 & $1.007^{\mathrm{c}}, 1.057^{\mathrm{d}}$ & 0.904 & 0.985 & 0.928 & 0.827 \\
\hline
\end{tabular}

Notes.

${ }^{a}$ For $\lambda \leqslant 72 \mu \mathrm{m}$

${ }^{\mathrm{b}}$ For $72 \mu \mathrm{m}<\lambda<100 \mu \mathrm{m}$.

${ }^{\mathrm{c}}$ For $100 \mu \mathrm{m} \leqslant \lambda<142 \mu \mathrm{m}$.

${ }^{\mathrm{d}}$ For $\lambda \geqslant 142 \mu \mathrm{m}$.

We follow the line flux measurement method of Lee et al. (2013) rather than that of Green et al. (2013). In order to correct for the extended nature of line emission, Green et al. (2013) derived an equation for line flux as a function of wavelength using strong $\mathrm{CO}$ lines and applied the equation to all species, except for [O I]. However, the emission lines of different species are distributed differently. In addition, three of our sources are mispointed, so the line emission distributes over multiple spaxels. In particular, the atomic lines are spatially distinct from the molecular lines in our targets; the atomic line emission is extended beyond the PACS point-spread function, but molecular line emission is mostly compact at similar wavelengths. To solve this problem, we measured fluxes of the extended (or mispointed) line emission by calculating the equivalent width over multiple spaxels, over which the line emission distributes, as explained in the Appendix of Lee et al. (2013). However, the key concept of flux measurement in Lee et al. (2013) is the same as that in Green et al. (2013). Both combine two data reductions done with two different Herschel Interactive Processing Environment (HIPE) versions (6.1, circa 2010-2011 and 8.1, circa 2012-2013) to produce reliable absolute flux calibration as well as the best signal-to-noise ratio $(\mathrm{S} / \mathrm{N})$; for line fluxes, the equivalent width calculated from the HIPE 8.1 reduction (with a higher $\mathrm{S} / \mathrm{N}$ ) is multiplied by the local continuum extracted from the whole 25 spaxels of the HIPE 6.1 reduction (with more accurate absolute flux calibration).

We improved upon the reduction used in Green et al. (2013) by applying correction factors to the blue and red spectra in order to align the different orders smoothly around $100 \mu \mathrm{m}$. We calculated the correction factors using the PACS 70 and $160 \mu \mathrm{m}$ photometric data, measured with the same aperture size as the PACS field of view because the continuum spectra are extracted from all 25 spaxels. The SEDs were convolved with the PACS wideband response functions to compare with actual photometric data. Correction factors were found separately for the blue and red spectra and listed in Table 2.

The flux of each transition of each source is listed in Table 3. The line detection criterion was $\mathrm{S} / \mathrm{N} \geqslant 3$ from the Gaussian line fitting. The errors presented in Table 3 are total flux errors calculated from the error propagation equation in the Appendix of Lee et al. (2013). However, the significance of line detection is decided by the rms noise of the baseline local to the line. In some sources, although the higher excitation lines are detected, the lower excitation CO lines are listed as non-detections because of their higher noise levels. In addition, some of the $\mathrm{H}_{2} \mathrm{O}$ and CO lines detected by Karska et al. (2013) were not detected in our observations. This apparent discrepancy in line detection is caused by the two different observing modes; the range scan mode used in this work accepts lower sensitivities to gain the full coverage of the PACS wavelength region, whereas the linescan mode from Karska et al. (2013) gains better spectral resolution 


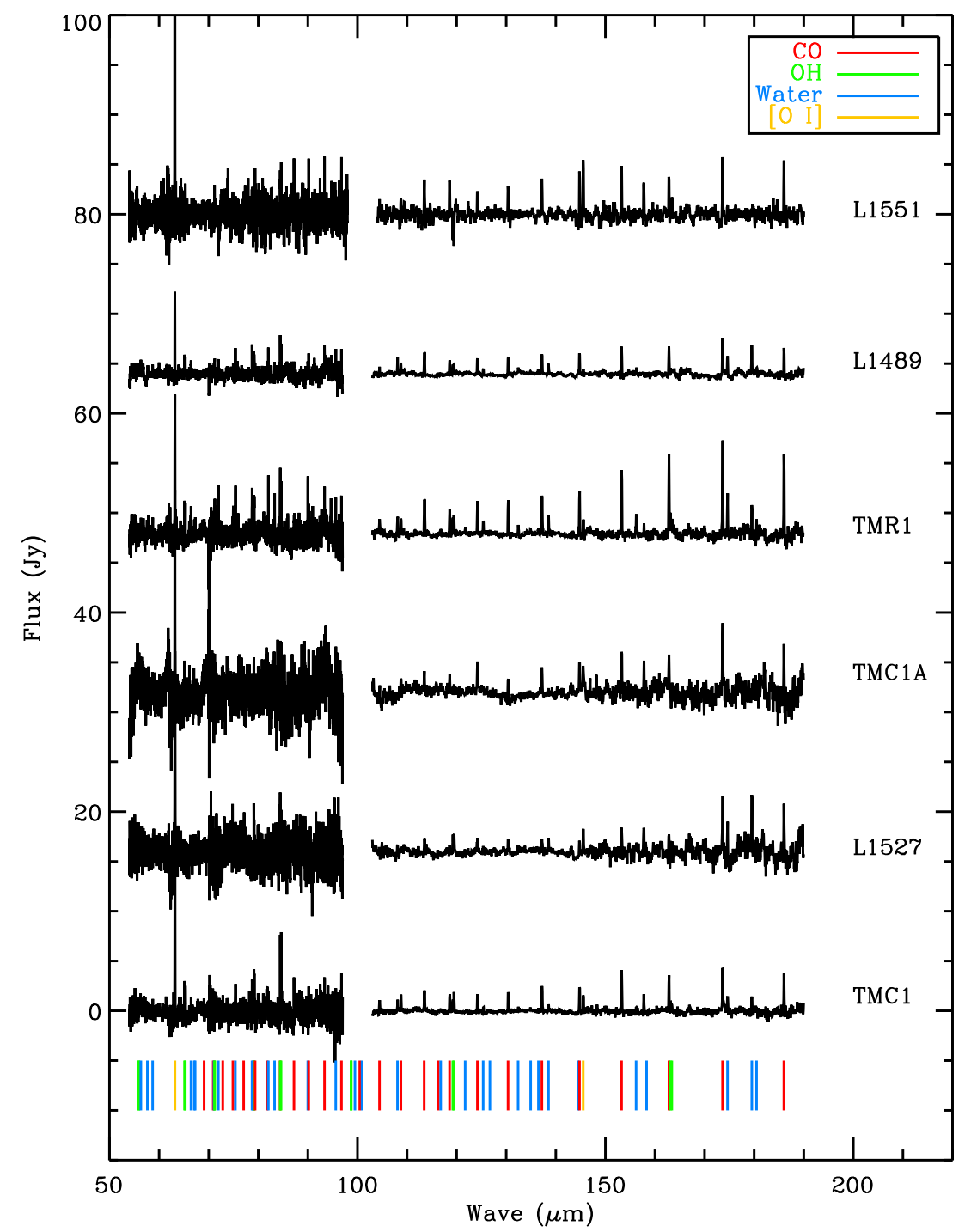

Figure 1. PACS continuum subtracted spectra of six Taurus sources. The spectra are extracted from the central spaxel in order to show lines with better $\mathrm{S} / \mathrm{Ns}$. However, for flux measurements, spectra were extracted from multi-spaxels over which emission was detected (check the text). From bottom to top, sources are arranged as their bolometric luminosities increase. (The fluxes of TMC1-A, L1527, and TMC1 have been doubled to show their lines better.)

(A color version of this figure is available in the online journal.)
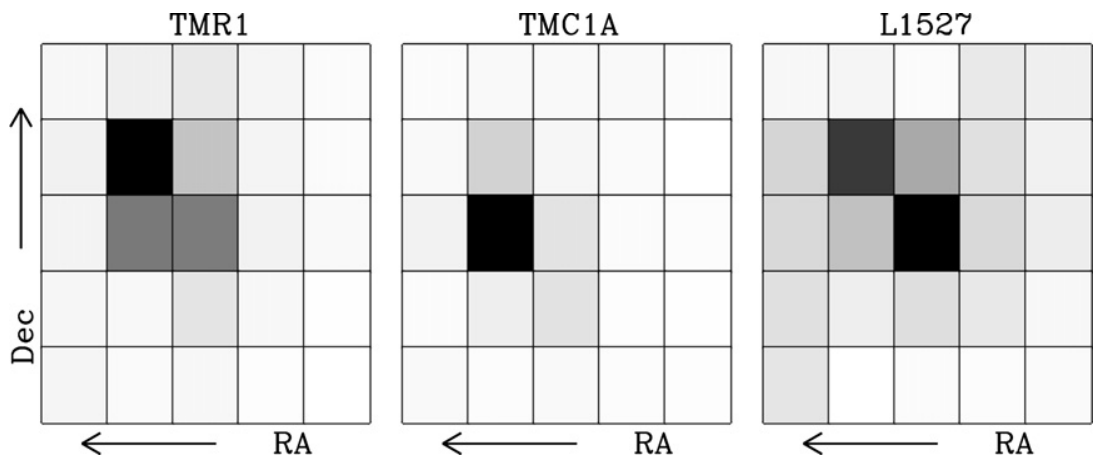

Figure $2.5 \times 5$ cube images of mispointed sources. Fluxes are integrated from $67.5 \mu \mathrm{m}$ to $68.5 \mu \mathrm{m}$ and decrease from black to white in the images.

and sensitivity over a very narrow wavelength range. In addition, the $\mathrm{CO} J=14-13$ flux is systematically lower than the $\mathrm{CO} J=$ 15-14 flux for all sources as listed in Table 3 because the level population of $J=15$ is greater than that of $J=14$ at the gas temperature above $250 \mathrm{~K}$, which is the case in our sources (see Section 4).

\section{DETECTED LINE TRANSITIONS}

\subsection{Molecular and Atomic Lines}

As shown in Figure 1 and Table 3, L1489, TMR1, and TMC1 have very rich emission line spectra. In contrast to previous studies (e.g., Nisini 2002; Karska et al. 2013), these sources do 
Table 3

Line Fluxes $\left(10^{-18} \mathrm{~W} \mathrm{~m}^{-2}\right)$

\begin{tabular}{|c|c|c|c|c|c|c|c|c|c|}
\hline Species & Transition & $\begin{array}{c}E_{u} \\
(\mathrm{~K})\end{array}$ & $\begin{array}{c}\lambda \\
(\mu \mathrm{m})\end{array}$ & L1489 & L1551-IRS5 & TMR1 & TMC1-A & L1527 & $\mathrm{TMC} 1$ \\
\hline \multirow[t]{19}{*}{$\mathrm{CO}$} & $36-35$ & 3668.78 & 72.84 & $\ldots$ & $\ldots$ & $31 \pm 14$ & $\ldots$ & $\ldots$ & $18 \pm 8$ \\
\hline & $35-34$ & 3471.27 & 74.89 & $\ldots$ & $\ldots$ & $133 \pm 45$ & $\ldots$ & $\ldots$ & $\ldots$ \\
\hline & $34-33$ & 3279.15 & 77.06 & $38 \pm 14$ & $\ldots$ & $\ldots$ & $\ldots$ & $\ldots$ & $28 \pm 8$ \\
\hline & $33-32$ & 3092.45 & 79.36 & $25 \pm 10$ & $\ldots$ & $77 \pm 23$ & $\ldots$ & $\ldots$ & $\ldots$ \\
\hline & $32-31$ & 2911.15 & 81.81 & $37 \pm 16$ & $\ldots$ & $72 \pm 27$ & $\ldots$ & $\ldots$ & $33 \pm 9$ \\
\hline & $30-29$ & 2564.83 & 87.19 & $52 \pm 23$ & $126 \pm 55$ & $82 \pm 28$ & $\ldots$ & $\ldots$ & $23 \pm 9$ \\
\hline & $29-28$ & 2399.82 & 90.16 & $\ldots$ & $\ldots$ & $70 \pm 26$ & $\ldots$ & $\ldots$ & $\ldots$ \\
\hline & $28-27$ & 2240.24 & 93.35 & $\ldots$ & $106 \pm 36$ & $90 \pm 37$ & $\ldots$ & $\ldots$ & $47 \pm 14$ \\
\hline & $25-24$ & 1794.23 & 104.45 & $65 \pm 23$ & $\ldots$ & $130 \pm 41$ & $50 \pm 17$ & $\ldots$ & $69 \pm 22$ \\
\hline & $24-23$ & 1656.47 & 108.76 & $77 \pm 22$ & $\ldots$ & $111 \pm 35$ & $55 \pm 24$ & $\ldots$ & $51 \pm 15$ \\
\hline & $22-21$ & 1397.38 & 118.58 & $87 \pm 25$ & $332 \pm 97$ & $158 \pm 45$ & $51 \pm 16$ & $53 \pm 20$ & $73 \pm 21$ \\
\hline & $21-20$ & 1276.05 & 124.19 & $112 \pm 32$ & $255 \pm 75$ & $175 \pm 50$ & $82 \pm 23$ & $129 \pm 41$ & $75 \pm 22$ \\
\hline & $20-19$ & 1160.20 & 130.37 & $117 \pm 33$ & $268 \pm 92$ & $178 \pm 51$ & $52 \pm 19$ & $73 \pm 28$ & $71 \pm 20$ \\
\hline & 19-18 & 1049.84 & 137.20 & $121 \pm 34$ & $259 \pm 73$ & $197 \pm 58$ & $62 \pm 19$ & $70 \pm 24$ & $106 \pm 31$ \\
\hline & $18-17$ & 944.97 & 144.78 & $123 \pm 34$ & $282 \pm 80$ & $186 \pm 55$ & $62 \pm 17$ & $67 \pm 21$ & $95 \pm 29$ \\
\hline & $17-16$ & 845.59 & 153.27 & $108 \pm 33$ & $246 \pm 72$ & $226 \pm 67$ & $56 \pm 19$ & $91 \pm 30$ & $100 \pm 31$ \\
\hline & $16-15$ & 751.72 & 162.81 & $113 \pm 32$ & $192 \pm 63$ & $232 \pm 66$ & $43 \pm 13$ & $60 \pm 26$ & $90 \pm 27$ \\
\hline & $15-14$ & 663.35 & 173.63 & $131 \pm 37$ & $383 \pm 115$ & $251 \pm 73$ & $69 \pm 20$ & $103 \pm 40$ & $117 \pm 33$ \\
\hline & 14-13 & 580.49 & 186.00 & $98 \pm 28$ & $248 \pm 74$ & $209 \pm 60$ & $47 \pm 15$ & $88 \pm 30$ & $86 \pm 25$ \\
\hline \multirow[t]{13}{*}{$\mathrm{OH}$} & $\frac{1}{2}, \frac{9}{2}-\frac{1}{2}, \frac{7}{2}$ & 875.10 & 55.89 & $\ldots$ & $\ldots$ & $\ldots$ & $\ldots$ & $\ldots$ & $18 \pm 5$ \\
\hline & & 875.10 & 55.95 & $\ldots$ & $\ldots$ & $\ldots$ & $\ldots$ & $\ldots$ & $36 \pm 10$ \\
\hline & $\frac{3}{2}, \frac{9}{2}-\frac{3}{2}, \frac{7}{2}$ & 512.10 & 65.13 & $140 \pm 40$ & $\ldots$ & $257 \pm 75$ & $\ldots$ & $\ldots$ & $94 \pm 28$ \\
\hline & & 510.90 & 65.28 & $81 \pm 23$ & $\ldots$ & $170 \pm 56$ & $\ldots$ & $\ldots$ & $93 \pm 28$ \\
\hline & $\frac{1}{2}, \frac{7}{2}-\frac{1}{2}, \frac{5}{2}$ & 617.60 & 71.17 & $46 \pm 14^{b}$ & $\ldots$ & $150 \pm 46$ & $74 \pm 25^{\mathrm{a}}$ & $\ldots$ & $\ldots$ \\
\hline & & 617.90 & 71.22 & $46 \pm 14$ & $\ldots$ & $80 \pm 32$ & $74 \pm 25$ & $\ldots$ & $\ldots$ \\
\hline & $\frac{1}{2}, \frac{1}{2}-\frac{3}{2}, \frac{3}{2}$ & 181.90 & 79.12 & $75 \pm 24$ & $\ldots$ & $147 \pm 51$ & $51 \pm 21$ & $119 \pm 37^{\mathrm{a}}$ & $64 \pm 25$ \\
\hline & & 181.70 & 79.18 & $74 \pm 28$ & $\ldots$ & $139 \pm 46$ & $\ldots$ & $119 \pm 37$ & $59 \pm 16$ \\
\hline & $\frac{3}{2}, \frac{7}{2}-\frac{3}{2}, \frac{5}{2}$ & 290.50 & 84.60 & $71 \pm 27$ & $122 \pm 39$ & $193 \pm 58$ & $\ldots$ & $\ldots$ & $105 \pm 33$ \\
\hline & $\frac{3}{2}, \frac{5}{2}-\frac{3}{2}, \frac{3}{2}$ & 120.70 & 119.23 & $73 \pm 22$ & $-260 \pm 77$ & $99 \pm 28$ & $\ldots$ & $113 \pm 32$ & $49 \pm 14$ \\
\hline & & 120.50 & 119.44 & $78 \pm 23$ & $-408 \pm 116$ & $118 \pm 33$ & $\ldots$ & $160 \pm 51$ & $70 \pm 20$ \\
\hline & $\frac{1}{2}, \frac{3}{2}-\frac{1}{2}, \frac{1}{2}$ & 270.20 & 163.12 & $23 \pm 9$ & $\ldots$ & $38 \pm 11$ & $\ldots$ & $\ldots$ & $25 \pm 8$ \\
\hline & & 269.80 & 163.40 & $24 \pm 10$ & $104 \pm 45$ & $\ldots$ & $\ldots$ & $\ldots$ & $22 \pm 10$ \\
\hline \multirow[t]{14}{*}{$\mathrm{p}-\mathrm{H}_{2} \mathrm{O}$} & $4_{31}-3_{22}$ & 552.30 & 56.33 & $94 \pm 33$ & $\ldots$ & $73 \pm 24$ & $\ldots$ & $\ldots$ & $\ldots$ \\
\hline & $4_{22}-313$ & 454.30 & 57.64 & $\ldots$ & $\ldots$ & $106 \pm 32$ & $\ldots$ & $\ldots$ & $\ldots$ \\
\hline & $3_{31}-2_{20}$ & 410.40 & 67.09 & $30 \pm 11$ & $\ldots$ & $70 \pm 29$ & $\ldots$ & $\ldots$ & $\ldots$ \\
\hline & $5_{24}-4_{13}$ & 598.80 & 71.07 & $53 \pm 17$ & $\ldots$ & $75 \pm 21$ & $\ldots$ & $\ldots$ & $\ldots$ \\
\hline & $7_{17}-6_{06}$ & 843.80 & 71.54 & $39 \pm 12$ & $\ldots$ & $123 \pm 42$ & $\ldots$ & $\ldots$ & $\ldots$ \\
\hline & $6_{15}-5_{24}$ & 781.10 & 78.93 & $\ldots$ & $\ldots$ & $111 \pm 37$ & $\ldots$ & $\ldots$ & $18 \pm 6$ \\
\hline & $6_{06}-5_{15}$ & 642.70 & 83.28 & $\ldots$ & $\ldots$ & $123 \pm 36$ & $\ldots$ & $\ldots$ & $17 \pm 7$ \\
\hline & $3_{22}-2_{11}$ & 296.80 & 89.99 & $36 \pm 14$ & $\ldots$ & $173 \pm 55$ & $\ldots$ & $\ldots$ & $24 \pm 7$ \\
\hline & $4_{04}-3_{13}$ & 319.50 & 125.35 & $54 \pm 17$ & $\ldots$ & $69 \pm 20$ & $\ldots$ & $49 \pm 17$ & $27 \pm 8$ \\
\hline & $3_{31}-3_{22}$ & 410.40 & 126.71 & $\ldots$ & $\ldots$ & $7 \pm 9$ & $\ldots$ & $\ldots$ & $\ldots$ \\
\hline & $3_{13}-2_{02}$ & 204.70 & 138.53 & $78 \pm 24$ & $\ldots$ & $85 \pm 25$ & $\ldots$ & $59 \pm 20$ & $23 \pm 7$ \\
\hline & $4_{13}-3_{22}$ & 396.40 & 144.52 & $27 \pm 11$ & $\ldots$ & $48 \pm 19$ & $\ldots$ & $\ldots$ & $\ldots$ \\
\hline & $3_{22}-3_{13}$ & 296.80 & 156.19 & $\ldots$ & $\ldots$ & $102 \pm 36$ & $\ldots$ & $\ldots$ & $\ldots$ \\
\hline & $3_{31}-4_{04}$ & 410.40 & 158.31 & $\cdots$ & $\cdots$ & $\ldots$ & $20 \pm 7$ & $\cdots$ & $\cdots$ \\
\hline \multirow[t]{16}{*}{$\mathrm{o}-\mathrm{H}_{2} \mathrm{O}$} & $10_{29}-10_{110}$ & 1861.30 & 55.84 & $48 \pm 16$ & $\ldots$ & $\ldots$ & $\ldots$ & $\ldots$ & $\ldots$ \\
\hline & $4_{32}-3_{21}$ & 550.40 & 58.70 & $56 \pm 20$ & $\ldots$ & $168 \pm 49$ & $\ldots$ & $\ldots$ & $20 \pm 6$ \\
\hline & $7_{16}-6_{25}$ & 1013.20 & 66.09 & $57 \pm 19$ & $\ldots$ & $84 \pm 31$ & $\ldots$ & $\ldots$ & $\ldots$ \\
\hline & $3_{30}-2_{21}$ & 410.70 & 66.44 & $62 \pm 23$ & $\ldots$ & $155 \pm 52$ & $\ldots$ & $\ldots$ & $20 \pm 6$ \\
\hline & $3_{30}-3_{03}$ & 410.70 & 67.27 & $\ldots$ & $\ldots$ & $81 \pm 28$ & $\ldots$ & $\ldots$ & $\ldots$ \\
\hline & $7_{07}-6_{16}$ & 843.50 & 71.95 & $65 \pm 22$ & $\ldots$ & $179 \pm 53$ & $\ldots$ & $\ldots$ & $22 \pm 6$ \\
\hline & $3_{21}-2_{12}$ & 305.30 & 75.38 & $89 \pm 25$ & $\ldots$ & $211 \pm 74$ & $\ldots$ & $\ldots$ & $54 \pm 18$ \\
\hline & $4_{23}-3_{12}$ & 432.20 & 78.74 & $83 \pm 25$ & $\ldots$ & $211 \pm 78$ & $\ldots$ & $\ldots$ & $36 \pm 12$ \\
\hline & $6_{16}-5_{05}$ & 643.50 & 82.03 & $77 \pm 31$ & $\ldots$ & $174 \pm 58$ & $\ldots$ & $40 \pm 14$ & $41 \pm 13$ \\
\hline & $2_{21}-1_{10}$ & 194.10 & 108.07 & $92 \pm 26$ & $\ldots$ & $148 \pm 47$ & $\ldots$ & $67 \pm 20$ & $40 \pm 12$ \\
\hline & $7_{34}-6_{43}$ & 1212.00 & 116.78 & $\ldots$ & $\ldots$ & $31 \pm 28$ & $\ldots$ & $\ldots$ & $\ldots$ \\
\hline & $4_{32}-4_{23}$ & 550.40 & 121.72 & $\ldots$ & $\ldots$ & $41 \pm 14$ & $\ldots$ & $\ldots$ & $\ldots$ \\
\hline & $4_{23}-4_{14}$ & 432.20 & 132.41 & $30 \pm 9$ & $\ldots$ & $54 \pm 16$ & $\ldots$ & $54 \pm 19$ & $\ldots$ \\
\hline & $5_{14}-5_{05}$ & 574.70 & 134.94 & $\ldots$ & $\ldots$ & $31 \pm 11$ & $\ldots$ & $\ldots$ & $\ldots$ \\
\hline & $3_{30}-3_{21}$ & 410.70 & 136.50 & $25 \pm 9$ & $\ldots$ & $34 \pm 15$ & $\ldots$ & $\ldots$ & $\ldots$ \\
\hline & $3_{03}-2_{12}$ & 196.80 & 174.63 & $76 \pm 25$ & $27 \pm 11$ & $102 \pm 30$ & $28 \pm 12$ & $79 \pm 24$ & $40 \pm 16$ \\
\hline
\end{tabular}


Table 3

(Continued)

\begin{tabular}{|c|c|c|c|c|c|c|c|c|c|}
\hline Species & Transition & $\begin{array}{c}E_{u} \\
(\mathrm{~K})\end{array}$ & $\begin{array}{c}\lambda \\
(\mu \mathrm{m})\end{array}$ & L1489 & L1551-IRS5 & TMR1 & TMC1-A & L1527 & TMC1 \\
\hline & $212-1_{01}$ & 114.40 & 179.53 & $103 \pm 30$ & $\ldots$ & $79 \pm 25$ & $\ldots$ & $117 \pm 35$ & $38 \pm 15$ \\
\hline & $221-212$ & 194.10 & 180.49 & $\ldots$ & $\ldots$ & $31 \pm 8$ & $\ldots$ & $\ldots$ & $\cdots$ \\
\hline$[\mathrm{O} \mathrm{I}]$ & ${ }^{3} \mathrm{P}_{1}-{ }^{3} \mathrm{P}_{2}$ & 227.71 & 63.18 & $462 \pm 132$ & $7147 \pm 2051$ & $784 \pm 237$ & $661 \pm 192$ & $900 \pm 266$ & $1049 \pm 309$ \\
\hline [O I] & ${ }^{3} \mathrm{P}_{0}-{ }^{3} \mathrm{P}_{1}$ & 326.58 & 145.53 & $28 \pm 11$ & $488 \pm 143$ & $48 \pm 14$ & $43 \pm 14$ & $82 \pm 30$ & $123 \pm 42$ \\
\hline$[\mathrm{C}$ II $]$ & ${ }^{2} \mathrm{P}_{\frac{3}{2}}-{ }^{2} \mathrm{P}_{\frac{1}{2}}$ & 91.21 & 157.741 & $\ldots$ & $545 \pm 161$ & $22 \pm 7$ & $32 \pm 9$ & $93 \pm 35$ & $119 \pm 35$ \\
\hline
\end{tabular}

Note. ${ }^{a}$ Unresolved doublet lines. Fluxes were divided by 2.
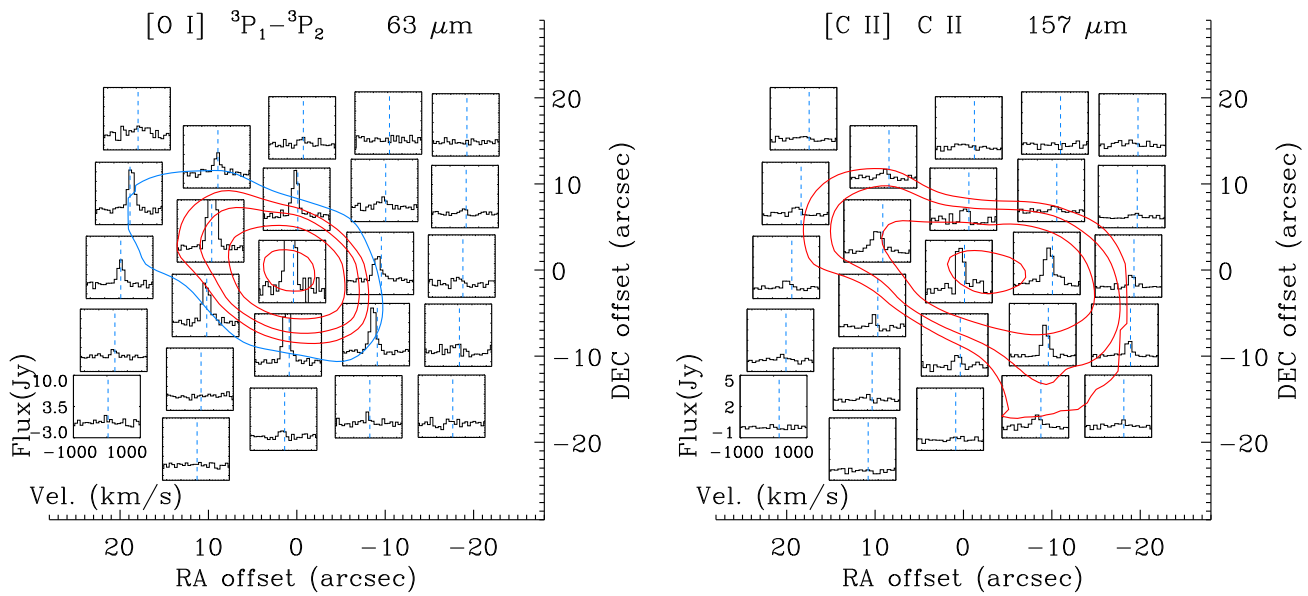

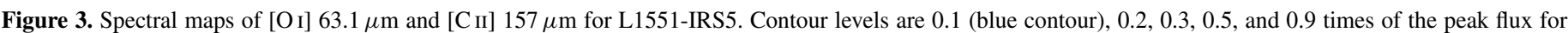
[O I] while $0.3,0.5,0.7$, and 0.9 of the peak flux for [C II]. We include one more blue contour in the [O I] map to make a better comparison with the [C II] map.

(A color version of this figure is available in the online journal.)

not have very high bolometric luminosities. TMC1, especially, has $L_{\text {bol }}$ less than $1 L_{\odot}$. The most luminous source among our targets is L1551-IRS5, but it does not show water lines except the $3_{03}-2_{12} 174.6 \mu \mathrm{m}$ transition. In addition, L1527, which has been known as a prominent outflow Class 0 source, shows neither high- $J\left(E_{\mathrm{u}}>1500 \mathrm{~K}\right) \mathrm{CO}$ transitions nor many water line transitions.

Toward all 6 sources, [O I] 63 and $145 \mu \mathrm{m}$ lines are detected. The [C II] $157 \mu \mathrm{m}$ line was also detected in all sources except L1489. These atomic lines have been considered as good tracers of the strength of the ISRF based on observations with the Infrared Satellite Observatory (ISO; Kaufman et al. 1999). However, it was difficult to separate the contribution from gas heated by the ISRF from the contribution by the source itself because of the poor spatial resolutions of ISO. Because Herschel has much better spatial resolution than did ISO (by a factor of 10), most Herschel observations have found that the [O I] emission distributes along the outflow direction (Karska et al. 2013; Dionatos et al. 2013; Lee et al. 2013), while [C II] emission generally shows no correlation with the source, indicating that it comes from the surface of the cloud.

Atomic emission is also extended in our sources; the [O I] $63 \mu \mathrm{m}$ emission is extended along the known outflow direction in all sources, but the $[\mathrm{C}$ II $] 157 \mu \mathrm{m}$ line is too weak to determine its extent in most sources. However, in L1551-IRS5 and TMC1, the $[\mathrm{CII}]$ line is strong and extended like the $[\mathrm{O} \mathrm{I}]$ line (see Figures 3 and 4) although their distributions differ somewhat. In TMC1-A, which is mispointed to the east only by one spaxel, the [OI] emission is elongated to the north, where the blueshifted outflow is located (Hogerheijde et al. 1998; Narayanan et al. 2012), as seen in Figure 5. The redshifted outflow located to the south in TMC1-A is very weak compared to the blueshifted component (Hogerheijde et al. 1998; Narayanan et al. 2012). Unlike the atomic emission, almost all molecular transitions are compact and peaked on the continuum source (see Figure 27 of Green et al. 2013); the mispointed sources do not seem to have extended emission structure either since the displacement of emission does not change with wavelength.

No O and B type massive young stars exist near the Taurus cloud to illuminate the large cloud structure. Therefore, these sources might be the best places to study the [O I] and [C II] emission directly related to protostars themselves. Indeed, in the Taurus sources, the [C II] emission is not diffuse and is very well correlated spatially with the [OI] emission, which distributes along the outflow direction (see Figures 3 and 4) in L1551-IRS5 and TMC1. Karska et al. (2013) also pointed out that the $[\mathrm{C}$ II $]$ emission is extended along the outflow direction in TMC1, and Goicoechea et al. (2012) showed a similar trend in Serpens SMM1. This might indicate that the main heating source of the atomic gas is the fast J-type shock, which can produce UV photons, and thus, dissociate $\mathrm{H}_{2} \mathrm{O}$ and $\mathrm{CO}$ resulting in high abundances of $\mathrm{O}$ and $\mathrm{C}^{+}$(Snell et al. 2005). Alternatively, the atomic line emission may be produced along the outflow cavity wall, where high-energy photons can penetrate to dissociate molecules to $\mathrm{O}$ and $\mathrm{C}^{+}$(Mamon et al. 1988). In the latter case, a $\mathrm{C}$ shock should still exist along the cavity wall to explain the broad component of molecular emission detected by HIFI (Kristensen et al. 2012). 

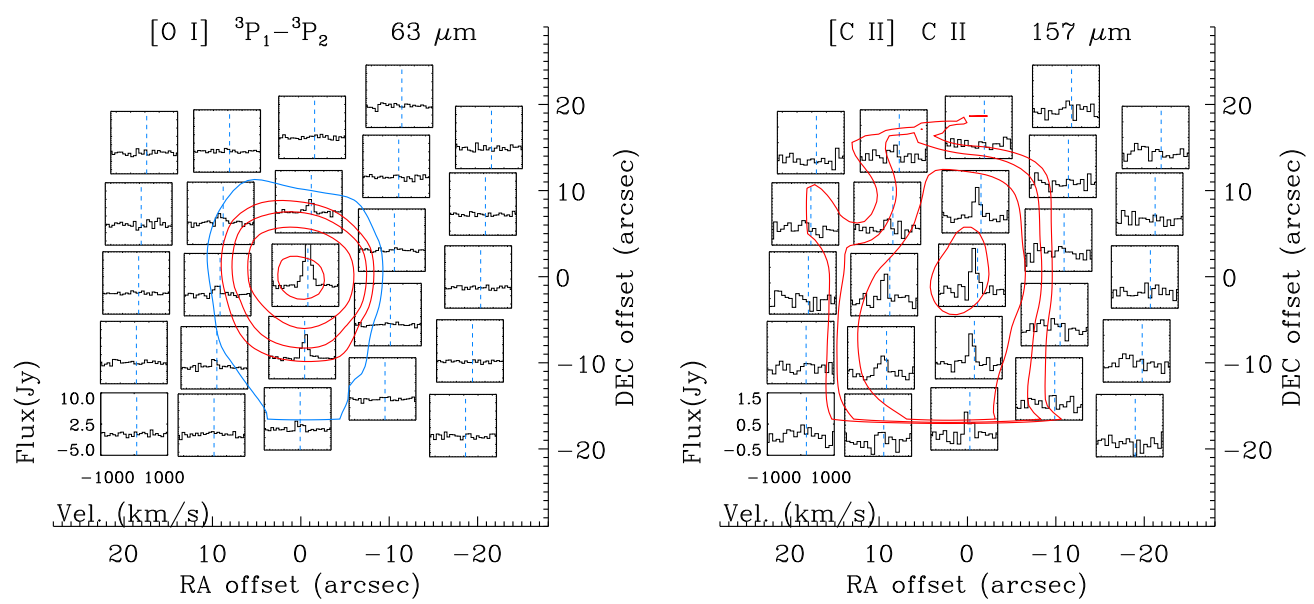

Figure 4. Spectral maps of [O I] $63.1 \mu \mathrm{m}$ and [C II] $157 \mu \mathrm{m}$ for TMC1. Contour levels are the same as Figure 3.

(A color version of this figure is available in the online journal.)

Table 4

Luminosities

\begin{tabular}{lccccccc}
\hline \hline & Species & L1489 & L1551-IRS5 & TMR1 & TMC1-A & L1527 & TMC1 \\
\hline \multirow{2}{*}{ Lines } & $\mathrm{CO}$ & $7.9 \pm 2.4$ & $16.3 \pm 5.0$ & $15.7 \pm 4.9$ & $3.8 \pm 1.3$ & $4.4 \pm 1.6$ & $6.6 \pm 2.0$ \\
$\left(10^{-4} L_{\odot}\right)$ & $\mathrm{OH}$ & $4.4 \pm 1.4$ & $1.4 \pm 0.5$ & $8.4 \pm 2.7$ & $1.2 \pm 0.4$ & $3.1 \pm 1.0$ & $3.9 \pm 1.2$ \\
& $\mathrm{H}_{2} \mathrm{O}$ & $7.7 \pm 1.9$ & $0.2 \pm 0.07$ & $18.0 \pm 4.4$ & $0.3 \pm 0.08$ & $2.8 \pm 0.7$ & $2.6 \pm 0.7$ \\
& {$[\mathrm{O}$ I $]$} & $3.0 \pm 0.9$ & $45.9 \pm 13.2$ & $5.0 \pm 1.5$ & $4.2 \pm 1.3$ & $5.9 \pm 1.8$ & $7.1 \pm 2.1$ \\
Total FIR line $^{\mathrm{a}}\left(L_{\text {line }}\right)\left(10^{-4} L_{\odot}\right)$ & {$[\mathrm{C} \mathrm{II}]$} & $\ldots$ & $3.3 \pm 1.0$ & $0.1 \pm 0.04$ & $0.2 \pm 0.05$ & $0.6 \pm 0.2$ & $0.7 \pm 0.2$ \\
\hline FIR continuum $^{\mathrm{b}}\left(L_{\text {cont }}\right)\left(L_{\odot}\right)$ & & 23.0 & 67.0 & 47.3 & 9.8 & 16.8 \\
\hline
\end{tabular}

Notes.

${ }^{\text {a }} L_{\text {line }}=L_{\mathrm{CO}}+L_{\mathrm{OH}}+L_{\mathrm{H}_{2} \mathrm{O}}+L_{[\mathrm{O} \mathrm{I}]}+L_{[\mathrm{C} \text { II }]}$.

${ }^{\mathrm{b}} L_{\text {cont }}=4 \pi d^{2} \int_{\mathrm{PACS}} f_{\nu} d \nu, d=140 \mathrm{pc}$. We integrated the flux density from $55 \mu \mathrm{m}$ to $190 \mu \mathrm{m}$ for $L_{\text {cont }}$.

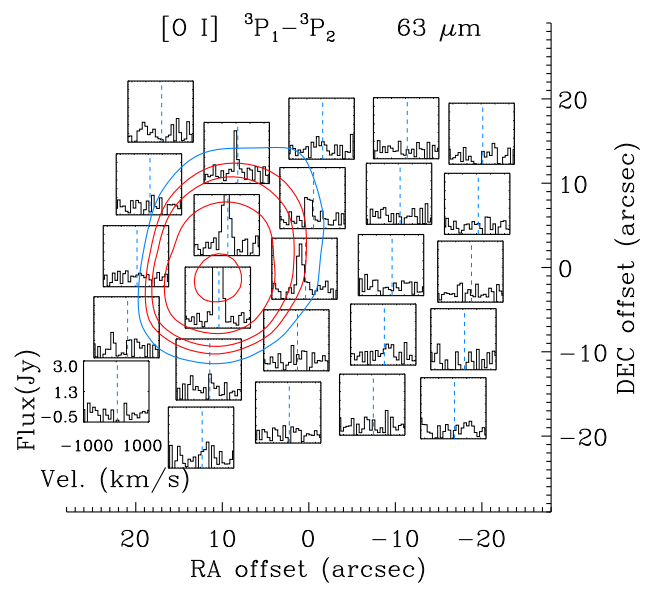

Figure 5. Spectral map of [OI] $63.1 \mu \mathrm{m}$ for TMC1-A. Contour levels are the same as Figure 3(a).

(A color version of this figure is available in the online journal.)

\subsection{FIR Line and Continuum Luminosities}

The observed PACS line luminosity of each species for each source is listed in Table 4. The FIR continuum luminosity in the PACS range is also listed. The total line luminosity is $\sim 0.1$ to $1 \%$ of the FIR continuum luminosity. The dominant cooling channel for the protostellar envelope is the FIR dust continuum emission. The brightest source, L1551-IRS5, has the smallest percentage $(0.06 \%)$ of line luminosity while the faintest source,
TMC1 has the biggest percentage (1\%) of line luminosity. As seen in Figure 6, the total FIR line luminosities $\left(L_{\text {line }}\right)$ have no correlation with $L_{\text {bol }}$ (bottom panel), which indicates that the line excitation is not simply related to the current source luminosity. The Pearson correlation coefficient between $L_{\text {line }}$ and $L_{\mathrm{bol}}$ is 0.53 , and its $p$ value is 0.26 . The $p$ value shows the fractional chance that the correlation is not significant. Therefore, in our samples, the correlation between $L_{\text {line }}$ and $L_{\text {bol }}$ is not statistically significant. However, as expected for embedded sources, the FIR continuum luminosity $\left(L_{\text {cont }}\right)$ in the PACS range tightly correlates with $L_{\mathrm{bol}}(r=0.98$ with a $p$ value $=$ 0.0006; see the upper panel of Figure 6); we find the relation of $L_{\text {bol }}=2.55 L_{\text {cont }}^{0.99}$. Therefore, the FIR continuum luminosity can be used as an accurate tracer of $L_{\mathrm{bol}}$ for embedded protostars. Dunham et al. (2008) showed that $L_{\text {bol }}$ can also be calculated from the flux at $70 \mu \mathrm{m}\left(L_{\mathrm{bol}} \propto F_{70}^{0.94}\right)$.

Figure 7 shows the relative contribution of each species to the total FIR line cooling. The CO luminosity is about $30 \%$ of the total FIR line luminosity for all sources. Except for L1551IRS5, the sum of $\mathrm{CO}$ and $\mathrm{OH}$ luminosity and the sum of $\mathrm{H}_{2} \mathrm{O}$ and [OI] luminosity each contribute half of the total FIR line luminosity. Except for L1551-IRS5, OH contributes about 20\% to the total FIR line luminosity.

The full spectral scans from the DIGIT program provide a check on conclusions from programs that observed only selected transitions to calculate the total line cooling. Karska et al. (2013) used the spectral mode of PACS to observe selected lines toward low-mass protostars, including all of our targets, but not L1551IRS5. In order to calculate the total CO luminosity in the PACS 


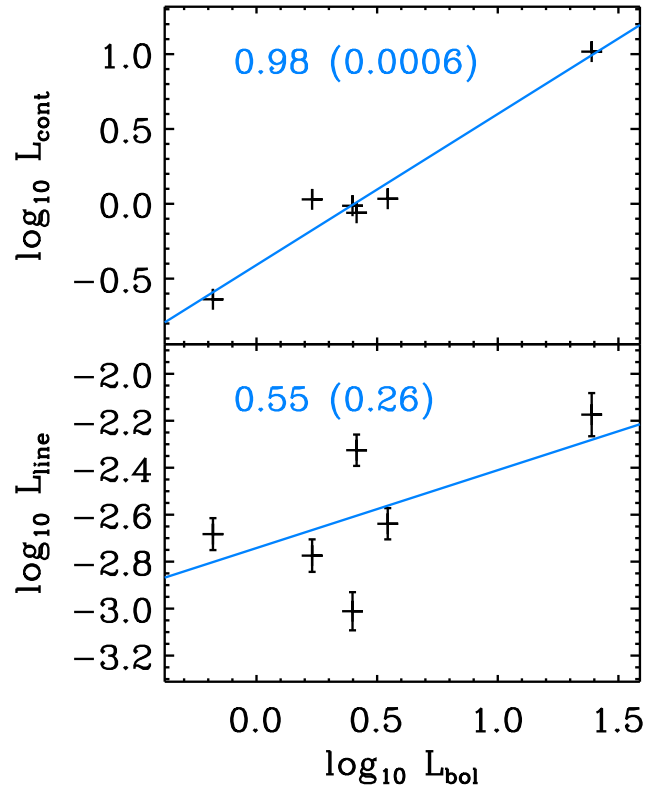

Figure 6. Correlations of continuum luminosity (top) and total FIR line luminosity (bottom) with bolometric luminosity. Luminosities are in the logarithmic scale. The FIR continuum luminosity has been calculated by integrating flux density from $55 \mu \mathrm{m}$ to $190 \mu \mathrm{m}$ with $d=140 \mathrm{pc}$. The correlation coefficients with $p$ values in parentheses are marked in the upper left of each box.

(A color version of this figure is available in the online journal.)

range, they used rotational temperatures from a subset of lines within their linescans while they used the ratios of twelve $\mathrm{H}_{2} \mathrm{O}$ and four $\mathrm{OH}$ line fluxes with respect to the total $\mathrm{H}_{2} \mathrm{O}$ and $\mathrm{OH}$ fluxes in the full PACS range for NGC 1333-IRAS4B and Serpens SMM1 to calculate the total PACS line luminosity of $\mathrm{H}_{2} \mathrm{O}$ and $\mathrm{OH}$ (see Figure 8 and Table 4 of Karska et al. 2013). Their results are not completely consistent with our results, based on the full spectral scans. For example, for TMR1, water contributes $\sim 40 \%$ to the total FIR line luminosity in our calculation, but only $\sim 20 \%$ in Karska et al. (2013). In addition, for TMC1-A, there is no contribution by water to the FIR line luminosity according to Karska et al. (2013) (because of no detection for the selected lines) while our observation shows that $\sim 5 \%$ of the FIR line luminosity is caused by water. On the other hand, for L1527, Karska et al. (2013) overestimated the contribution of water line to the total FIR line luminosity $(\sim 30 \%)$ by a factor of two compared to our calculation. As a whole, we calculated the percentage difference between our results and Karska et al. (2013) for each species among five sources; the biggest difference appears in water $(\sim 60 \%)$. The average difference for $\mathrm{CO}, \mathrm{OH}$, and [O I] are about $20 \%$. The average difference over four species for each source is about $65 \%$ for TMC1-A, 30\% for L1527 and TMR1, and about 20\% for L1489 and TMC1. These differences suggest that the flux ratio between selected lines and the full array of lines (especially for $\mathrm{H}_{2} \mathrm{O}$ ) in specific sources is not applicable to all sources.

The obvious result from our analysis is that water is not the most dominant coolant in most Taurus cloud sources; only in L1489 and TMR1, about $40 \%$ of FIR line luminosity is attributed to water lines while the water line luminosity is less than $20 \%$ of the total FIR line luminosity for the other four sources. For L1551-IRS5, about $70 \%$ of the FIR line luminosity is contributed by [O I] with little contribution by $\mathrm{H}_{2} \mathrm{O}$ and $\mathrm{OH}$, indicative of nearly complete dissociation of $\mathrm{H}_{2} \mathrm{O}$ and $\mathrm{OH}$. The differing contributions of each species to the total FIR line luminosity suggests different detailed heating and chemistry in each source.

\section{LINE ANALYSIS}

\subsection{Rotation Diagrams}

The simplest analysis of the rotational transitions of each molecule detected with PACS is the rotation diagram, where lines are assumed optically thin, and the same excitation temperature is applied to a range of level populations. Green et al. (2013) and Lee et al. (2013) have described the construction

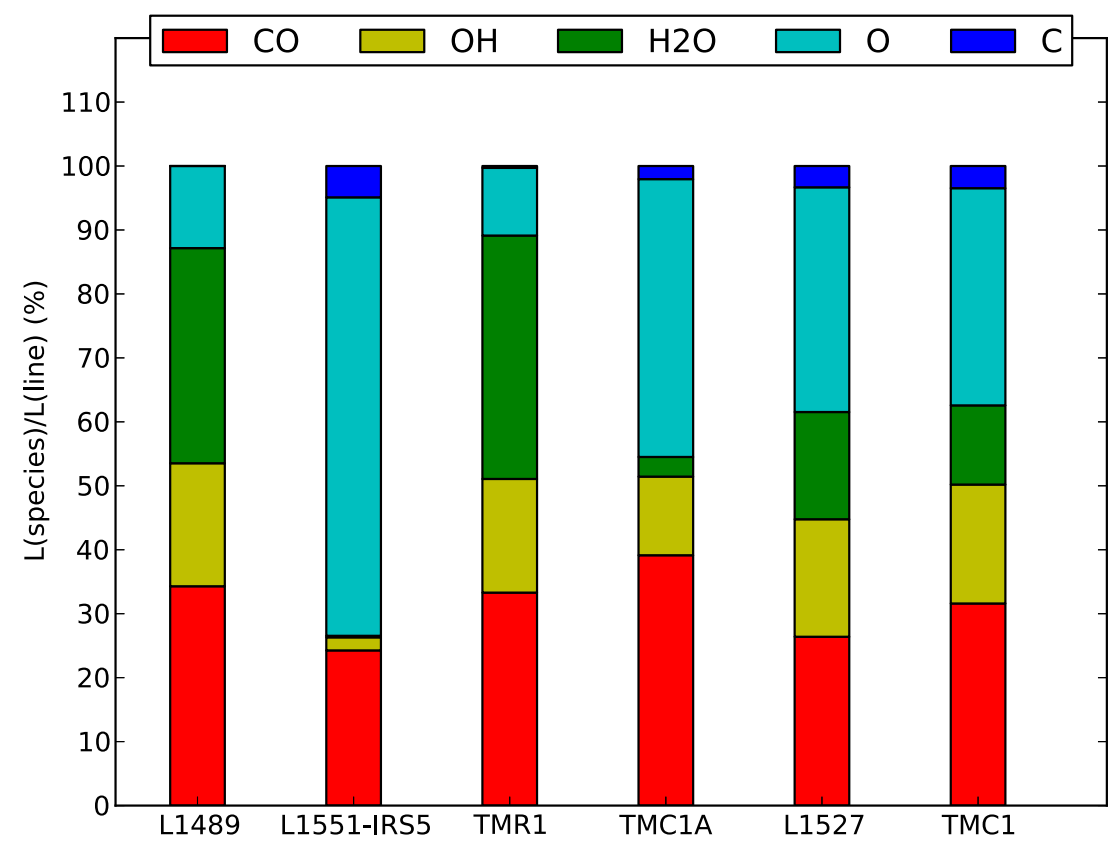

Figure 7. Relative contribution of each species to the total FIR line cooling for each source.

(A color version of this figure is available in the online journal.) 
Table 5

Results of the CO Rotation Diagram

\begin{tabular}{llcc}
\hline \hline Source & Component & $\begin{array}{c}T_{\text {rot }} \\
(\mathrm{K})\end{array}$ & $\begin{array}{c}\mathcal{N}(\mathrm{CO}) \\
\left(\times 10^{47}\right)\end{array}$ \\
\hline L1489 & Warm & $372 \pm 34$ & $18.6 \pm 5.2$ \\
& Hot & $754 \pm 446$ & $6.2 \pm 14.7$ \\
L1551-IRS5 & Warm & $394 \pm 59$ & $42.0 \pm 16.0$ \\
& Hot & $2198 \pm 8274$ & $458.9 \pm 1860.5$ \\
TMR1 & Warm & $351 \pm 30$ & $36.3 \pm 10.0$ \\
& Hot & $1117 \pm 371$ & $14.8 \pm 13.0$ \\
TMC1-A & Warm & $442 \pm 53$ & $8.5 \pm 2.6$ \\
L1527 & Warm & $361 \pm 60$ & $13.8 \pm 6.4$ \\
TMC1 & Warm & $363 \pm 32$ & $15.6 \pm 4.4$ \\
& Hot & $777 \pm 197$ & $5.0 \pm 4.8$ \\
\hline
\end{tabular}

Table 6

Results of the $\mathrm{OH}$ Rotation Diagram

\begin{tabular}{llrr}
\hline \hline Source & Component & \multicolumn{1}{c}{$\begin{array}{c}\text { rot } \\
(\mathrm{K})\end{array}$} & \multicolumn{1}{c}{$\begin{array}{c}\mathcal{N}(\mathrm{OH}) \\
\left(\times 10^{44}\right)\end{array}$} \\
\hline \multirow{2}{*}{ 1489 } & ${ }^{2} \Pi_{3 / 2}-{ }^{2} \Pi_{3 / 2}$ & $132 \pm 13$ & $8.7 \pm 2.4$ \\
& ${ }^{2} \Pi_{1 / 2}-{ }^{2} \Pi_{1 / 2}$ & $96 \pm 10$ & $7.5 \pm 4.3$ \\
TMR1 & ${ }^{2} \Pi_{3 / 2}-{ }^{2} \Pi_{3 / 2}$ & $148 \pm 17$ & $13.1 \pm 3.4$ \\
& ${ }^{2} \Pi_{1 / 2}-{ }^{2} \Pi_{1 / 2}$ & $110 \pm 14$ & $12.1 \pm 7.0$ \\
TMC1 & ${ }^{2} \Pi_{3 / 2}-{ }^{2} \Pi_{3 / 2}$ & $136 \pm 14$ & $7.4 \pm 2.0$ \\
& ${ }^{2} \Pi_{1 / 2}-{ }^{2} \Pi_{1 / 2}$ & $111 \pm 7$ & $7.7 \pm 3.0$ \\
\hline
\end{tabular}

of rotation diagrams with DIGIT data in detail. We plot the log of total number of molecules per degenerate sublevel $\left(\mathcal{N} / g_{\mathrm{J}}\right)$ versus the energy in the upper state, expressed in kelvin $\left(E_{\mathrm{u}}\right)$. As found in many other sources, the observed $\mathrm{CO}$ fluxes show a positive curvature in the rotation diagrams of four of our sources. Therefore, for L1489, L1551-IRS5, TMR1, and TMC1, we fitted the $\mathrm{CO}$ rotation diagram with two rotational temperatures with a break point at $E_{\mathrm{u}} \sim 1800 \mathrm{~K}$ as is typical from previous studies; the fitting results are not affected by moving the break point by $\Delta J \leqslant 2$ in either direction (Green et al. 2013). In L1551IRS5, only two transitions with $E_{\mathrm{u}} \geqslant 1800 \mathrm{~K}$ were detected, and thus the hot component is poorly constrained. For the other two sources, without detection of CO lines at $E_{\mathrm{u}}>1800 \mathrm{~K}$, we fitted only one temperature. The results are summarized in Table 5.

For the rotation diagrams with two temperatures, high- $J \mathrm{CO}$ lines $\left(E_{\mathrm{u}} \geqslant 1800 \mathrm{~K}\right)$ were fitted by a component $\geqslant 700 \mathrm{~K}$ (referred to as the "hot" component) while low- $J$ CO fluxes were fitted to $350 \sim 400 \mathrm{~K}$ (the "warm" component). The total number of emitting molecules, $\mathcal{N}(\mathrm{CO})$, is always greater for the warm component than the hot component, as found by Green et al. (2013) and Karska et al. (2013). The average ratio of $\mathcal{N}(\mathrm{CO})$ between warm and hot components is about 2.5 (with a standard deviation $\sigma$ of 0.2 ), which is close to the average ratio $(\sim 2.1$ with $\sigma \sim 0.7)$ in Karska et al. (2013) but much smaller than the average ratio $(\sim 8.5$ with $\sigma \sim 1.7)$ in Green et al. (2013) when only Taurus sources in those papers are included. However, if all WISH and DIGIT sources are considered, the average ratios are $\sim 6$ and $\sim 10$, respectively, indicating that Taurus sources have more hot $\mathrm{CO}$ components compared to YSOs in other star forming regions. We note that our line fluxes are more consistent with Karska et al. (2013) than Green et al. (2013).

The $\mathrm{OH}$ fluxes were fitted separately for lines in the $\Pi_{1 / 2}$ and $\Pi_{3 / 2}$ ladders, and the fluxes were fitted to $T_{\text {rot }}$ of $\sim 100 \mathrm{~K}$ with $\mathcal{N}(\mathrm{OH})$ of $\sim 10^{45}$. The results are summarized in Table 6 .
Table 7

Results of the $\mathrm{H}_{2} \mathrm{O}$ Rotation Diagram

\begin{tabular}{llcr}
\hline \hline Source & Component & $\begin{array}{c}T_{\text {rot }} \\
(\mathrm{K})\end{array}$ & $\begin{array}{r}\mathcal{N}\left(\mathrm{H}_{2} \mathrm{O}\right) \\
\left(\times 10^{44}\right)\end{array}$ \\
\hline L1489 & & $328 \pm 22$ & $7.4 \pm 1.1$ \\
& Ortho & $155 \pm 14$ & $5.0 \pm 1.5$ \\
TMR1 & Para & $191 \pm 12$ & $17.9 \pm 3.1$ \\
& Ortho & $177 \pm 15$ & $9.2 \pm 2.3$ \\
L1527 & Para & $119 \pm 11$ & $16.6 \pm 4.4$ \\
& Ortho & $131 \pm 75$ & $7.0 \pm 8.1$ \\
TMC1 & Para & $140 \pm 10$ & $2.8 \pm 0.7$ \\
& Ortho & $210 \pm 31$ & $2.2 \pm 0.7$ \\
\hline
\end{tabular}

If we fit both ladders altogether, the rotation temperature results in a value between two temperatures fitted separately. We also fitted $\mathrm{OH}$ fluxes only at $E_{\mathrm{u}}<400 \mathrm{~K}$ and found higher rotation temperatures $(\sim 200 \mathrm{~K})$. These higher rotational temperatures were seen in low-mass protostars in Karska et al. (2014) although intermediate mass protostars show much lower rotational temperatures. For L1551-IRS5, TMC1-A, and L1527, very few $\mathrm{OH}$ lines are detected so that rotation diagrams are not useful.

For water, we fitted $\mathrm{o}-\mathrm{H}_{2} \mathrm{O}$ (ortho-water) and $\mathrm{p}-\mathrm{H}_{2} \mathrm{O}$ (parawater) separately and derived $T_{\text {rot }}$ of $100-300 \mathrm{~K}$ with $\mathcal{N}\left(\mathrm{H}_{2} \mathrm{O}\right)$ of $10^{44}-10^{45}$ (see Table 7). L1551-IRS5 and TMC1-A have only one and two water line detected, respectively, so that rotation diagrams are not useful. The ortho-to-para ratio (OPR) derived from the total numbers of $\mathrm{H}_{2} \mathrm{O}$ molecules varies from source to source but is never greater than 2.5, indicative of a low temperature formation mechanism. In temperatures higher than $50 \mathrm{~K}$, the equilibrium value of the OPR is 3 (Mumma et al. 1987). For all sources except for L1527, the OPR is certainly smaller than 2.5 even if the uncertainties of the derived column densities are considered. A ratio of 2.5 suggests formation below $\sim 30 \mathrm{~K}$ (Mumma et al. 1987), much lower than their current rotational temperature. The water that we observe might have formed on cold grain surfaces and evaporated when the grains were heated by energetic photons. The water ice can be also sputtered by shock (Neufeld et al. 2014) or photodesorbed by UV photons (Lee et al. 2014). The timescale after evaporation (sputtering or photodesorption) should not be long enough to equilibrate the ratio with the gas temperature. Figure 8 presents the $\mathrm{CO}$, $\mathrm{H}_{2} \mathrm{O}$, and $\mathrm{OH}$ rotation diagrams of TMR1 as examples since TMR1 shows the richest line emission among our sources. The rotation diagrams for other sources can be found in the online supplementary material.

\subsection{LVG Models}

The assumptions of the rotation diagram analysis (optically thin lines and a single rotational temperature) may not hold for all PACS lines. Even if a single rotational temperature fits the data, it may not be equal to the true kinetic temperature of the gas. In addition, the rotation diagram cannot provide direct information on the density of the associated gas. Therefore, we adopted the non-LTE LVG code, RADEX (van der Tak et al. 2007) to model the intensities of the detected lines. The three parameters $\left(T_{\mathrm{K}}, n\left(\mathrm{H}_{2}\right)\right.$, and $N$ (molecule) $\left./ \Delta v\right)$ are used to determine the level population. We used the LAMDA database (Schoier et al. 2005) for molecular data. Because we do not know the actual solid angle of the source or the linewidth, we cannot convert intensity to line flux. Therefore, we applied a scaling 

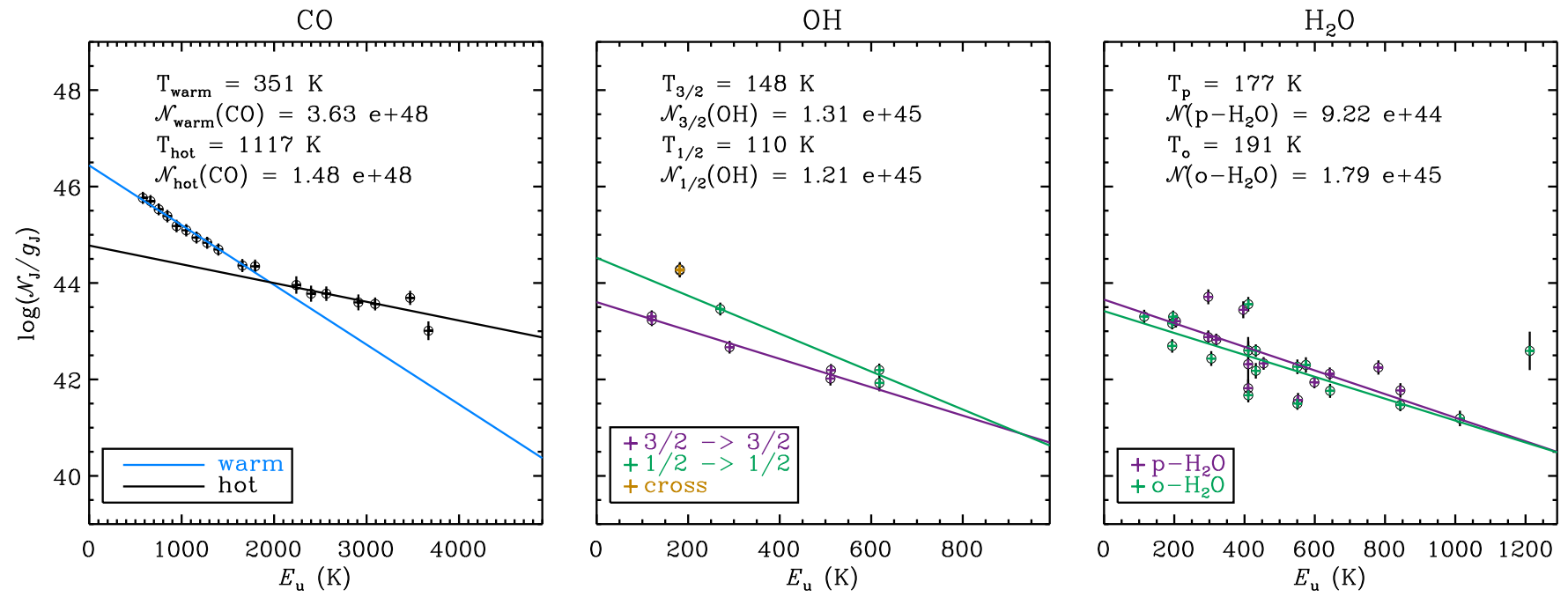

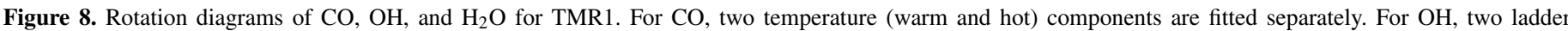

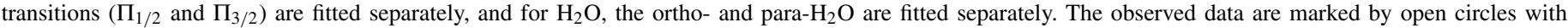
error bars.

(The complete figure set (6 images) and a color version of this figure are available in the online journal.)

Table 8

The Best-fit LVG Models of CO

\begin{tabular}{|c|c|c|c|c|c|c|c|}
\hline & & L1489 & L1551-IRS5 & TMR1 & TMC1-A & L1527 & TMC1 \\
\hline \multirow{5}{*}{1 comp. } & $T(\mathrm{~K})$ & $5.0(3)^{\mathrm{a}, \mathrm{b}}$ & $5.0(3)^{b}$ & $5.0(3)^{\mathrm{b}}$ & $4.0(3)$ & $5.0(3)^{b}$ & $5.0(3)^{\mathrm{b}}$ \\
\hline & $n\left(\mathrm{H}_{2}\right)\left(\mathrm{cm}^{-3}\right)$ & $3.2(4)$ & $3.2(4)$ & $1.8(4)$ & $1.0(5)$ & $5.6(4)$ & $1.0(3)$ \\
\hline & $N(\mathrm{CO}) / \Delta \mathrm{v}\left(\mathrm{cm}^{-2} /\left[\mathrm{km} \mathrm{s}^{-1}\right]\right)$ & $3.2(16)$ & $1.0(15)$ & $1.0(15)$ & $1.0(15)$ & $1.0(15)$ & $1.0(15)$ \\
\hline & Scale factor ${ }^{\mathrm{c}}$ & $1.2(0)$ & $1.0(2)$ & $1.4(2)$ & $1.1(1)$ & $1.8(1)$ & $1.5(3)$ \\
\hline & $\chi^{2}$ & 2.5 & 6.6 & 3.7 & 5.1 & 4.1 & 3.7 \\
\hline \multirow{4}{*}{ Power law } & $n\left(\mathrm{H}_{2}\right)\left(\mathrm{cm}^{-3}\right)$ & $1.0(5)$ & $3.2(5)$ & $3.2(6)$ & $5.6(5)$ & $5.6(5)$ & $1.8(6)$ \\
\hline & $p$ & 1.0 & 1.8 & 2.8 & 1.6 & 2.0 & 2.8 \\
\hline & Scale factor & $1.0(2)$ & $1.3(3)$ & $1.4(4)$ & $1.2(2)$ & $6.5(2)$ & $7.5(3)$ \\
\hline & $\chi^{2}$ & 2.6 & 5.3 & 2.1 & 4.0 & 3.1 & 2.4 \\
\hline
\end{tabular}

Notes.

a $a(\mathbf{b}) \equiv a \times 10^{b}$.

$\mathrm{b}$ This temperature is the highest temperature in the collisional rate.

${ }^{\mathrm{c}}$ Scale factor $=$ AREA $\left(\operatorname{arcsec}^{2}\right) \times$ FWHM $\left(\mathrm{km} \mathrm{s}^{-1}\right)$.

factor to models that gives the minimum $\chi^{2}$. The scaling factor is the product of emitting area and line width.

In the rotation diagrams, the $\mathrm{CO}$ gas seems to have two temperature components of 300-400 K and 700-1100 K in our samples (Table 5). However, Neufeld (2012) suggested that one gas component with a very high temperature $(>1000 \mathrm{~K})$ with a low $n\left(\mathrm{H}_{2}\right)$ of $10^{4} \sim 10^{5} \mathrm{~cm}^{-3}$ could generate a positively curved $\mathrm{CO}$ rotation diagram. They also pointed out that gas with a power-law temperature distribution can account for the observed curvature in rotation diagrams.

\subsection{1. $\mathrm{CO}$}

In our RADEX models of CO, we tested the case of a single gas component and also gas with a power-law temperature distribution $\left(d N \propto T^{-p} d T\right)$. The ranges of physical conditions tested were $50 \mathrm{~K} \leqslant T_{\mathrm{K}} \leqslant 5000 \mathrm{~K}, 10^{3} \mathrm{~cm}^{-3} \leqslant n\left(\mathrm{H}_{2}\right) \leqslant 10^{13} \mathrm{~cm}^{-3}$, and $10^{13} \mathrm{~cm}^{-2} \mathrm{~km}^{-1} \mathrm{~s}^{-1} \leqslant N(\mathrm{CO}) / \Delta v \leqslant 10^{20} \mathrm{~cm}^{-2} \mathrm{~km}^{-1} \mathrm{~s}^{-1}$ for the one-component model. The temperature range for the power-law model is 10 to $5000 \mathrm{~K}$, and the total $N(\mathrm{CO}) / \Delta v$ is fixed to $10^{15} \mathrm{~cm}^{-2} \mathrm{~km}^{-1} \mathrm{~s}^{-1}$, which satisfies the optically thin assumption and reduces the number of free parameters. The density range was $10^{3} \mathrm{~cm}^{-3} \leqslant n\left(\mathrm{H}_{2}\right) \leqslant 10^{13} \mathrm{~cm}^{-3}$, and the power index range was $0<p<4$. We calculated the collisional rates up to $5000 \mathrm{~K}$ by following Neufeld (2012) and Schoier et al. (2005). The best-fit models are summarized in Table 8.

For the one-component model, these sources prefer a subthermal solution with $n \leqslant 10^{5} \mathrm{~cm}^{-3}$ and $T=5000 \mathrm{~K}$, which is the highest temperature for available collision rates. Therefore, the temperature of this model is not constrained. If a higher temperature is available, it would fit the observations better with a lower density.

Therefore, if a $\mathrm{C}$ shock is responsible for these $\mathrm{CO}$ fluxes, the shock velocity must be $\geqslant 40 \mathrm{~km} \mathrm{~s}^{-1}$ in order to provide $T_{K} \geqslant 5000 \mathrm{~K}$ (Flower \& Pineau des Forêts 2010). The density derived from this model is the post-shock density. Therefore, if we consider the enhancement factor of density by shocks, the pre-shock density is smaller than the derived values by an order of magnitude (for $\mathrm{C}$ shocks) or more (for $\mathrm{J}$ shocks). The densities for the one-component models are mostly $\leqslant 10^{5} \mathrm{~cm}^{-3}$, which would imply pre-shock densities $\leqslant 10^{4} \mathrm{~cm}^{-3}$. If these are shocks into the cavity walls, they would need to be far from the source to get such low densities, but we observe compact emission. The alternative is low density molecular gas within the outflow cavity rather than on the walls. 

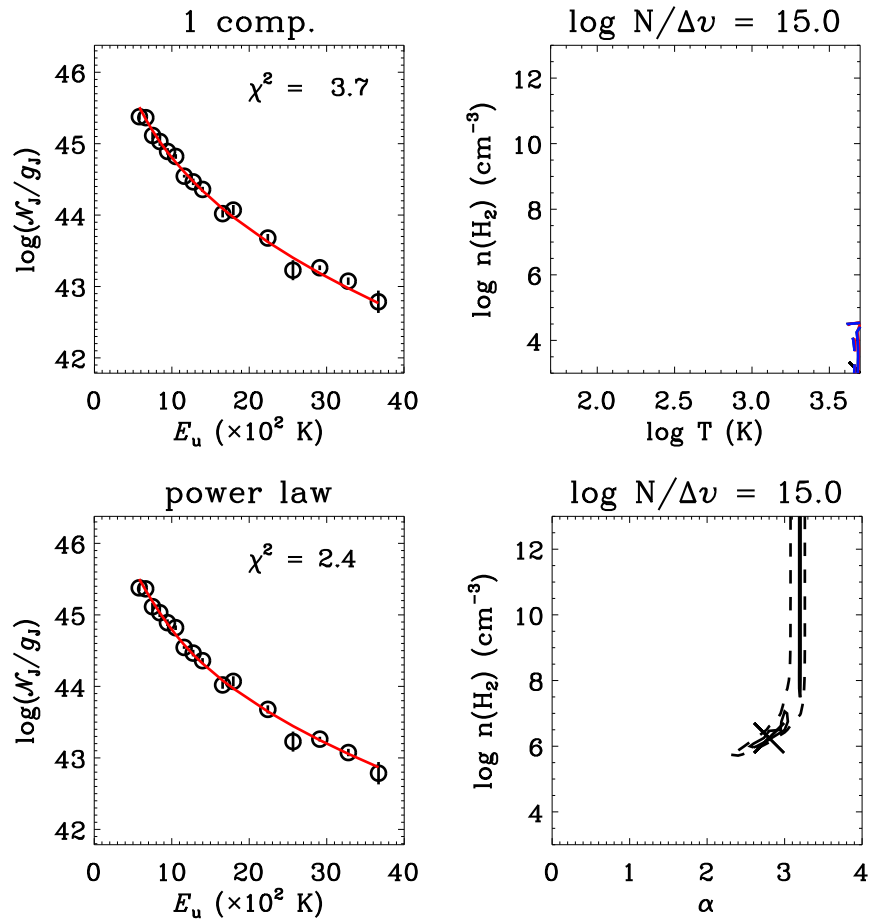

Figure 9. Best-fit RADEX models for CO lines (top panels for the onecomponent model and bottom panels for the power-law temperature distribution model) in TMC1. In rotation diagrams (left panels), circles with error bars are observed data, and the solid lines represent the best-fit models. The physical conditions for the models are summarized in Table 8 . The reduced $\chi^{2}$ is presented as contours in the $(n, T)$ space at the best-fit column density (right panels). The contours are 1.2 (solid line) and 1.5 (dashed line) times the best $\chi^{2}$ value (cross), which is presented in the rotation diagrams. For the onecomponent model (top right), the black contours represent $\chi^{2}$ for the best-fit model, but blue and red contours show $\chi^{2}$ for the models with lower and higher column densities, respectively, than the best-fit column density by a factor of 10. However, all these models collapse to the sub-thermal solution with a low density and the highest temperature available, so those different color contours are not distinguishable in the plot.

(The complete figure set ( 8 images) and a color version of this figure are available in the online journal.)

For the power-law model, our sources require a higher density $\left(1.0 \times 10^{5}\right.$ to $\left.3.2 \times 10^{6} \mathrm{~cm}^{-3}\right)$ with $p \sim 1-3$. The pre-shock density for the best-fit models of TMR 1 and TMC1 can be found at $\sim 1000 \mathrm{AU}$ for their envelope models (Kristensen et al. 2012) if a $\mathrm{C}$ shock is responsible for the $\mathrm{CO}$ emission. However, the pre-shock density for the best-fit model for L1489 can be found at the radius greater than $1000 \mathrm{AU}$ or within the outflow cavity.

The best $\mathrm{CO}$ models of TMC1 are presented in Figure 9. Figures for the RADEX models for other sources can be found in the online material. For the CO models, only line flux measurement errors are considered because we are the most interested in fitting the shape of the $\mathrm{CO}$ rotation diagrams. (For other molecules, we consider the total errors, which are dominated by the continuum calibration errors. The continuum calibration errors are assumed to be $20 \%$ of the continuum fluxes.) For TMC1 and TMR1, the power-law model fits the CO observations better than the single temperature model, although all other sources show similar reduced $\chi^{2}$ for the power-law and one-component models (see Table 8 ). Figure 10 shows the relative (left) and cumulative (right) contributions of different temperatures to the total flux for the best-fit powerlaw temperature model of TMC1. Most of flux comes from the gas with $T<1000 \mathrm{~K}$ for TMC1 and TMR1, where the best-fit density is greater than $10^{6} \mathrm{~cm}^{-3}$ with $p=2.8$.
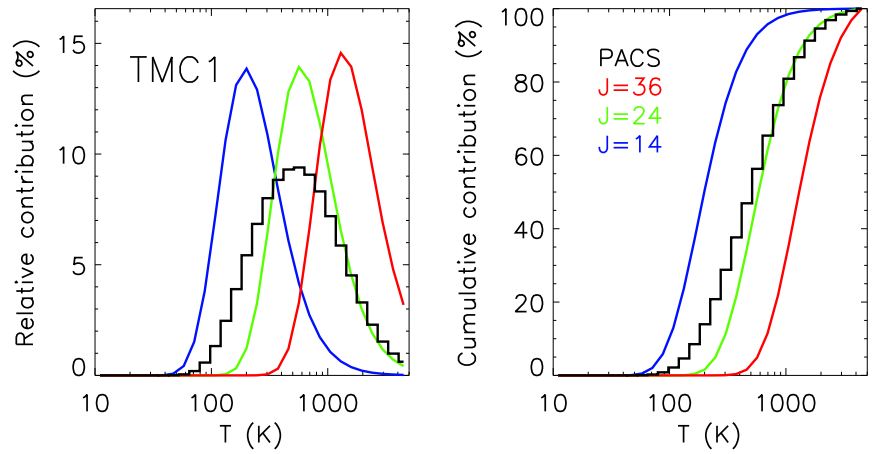

Figure 10. Relative (left) and cumulative (right) contribution of different temperatures to the total $\mathrm{CO}$ flux at the PACS wavelength range (black histogram) and fluxes of $J=14-13$ (blue line), $J=24-23$ (green line), and $J=$ $36-35$ (red line) in the best-fit power-law model for TMC1. Most $(\sim 70 \%)$ of the total CO flux comes from the gas with $T<1000 \mathrm{~K}$.

(A color version of this figure is available in the online journal.)
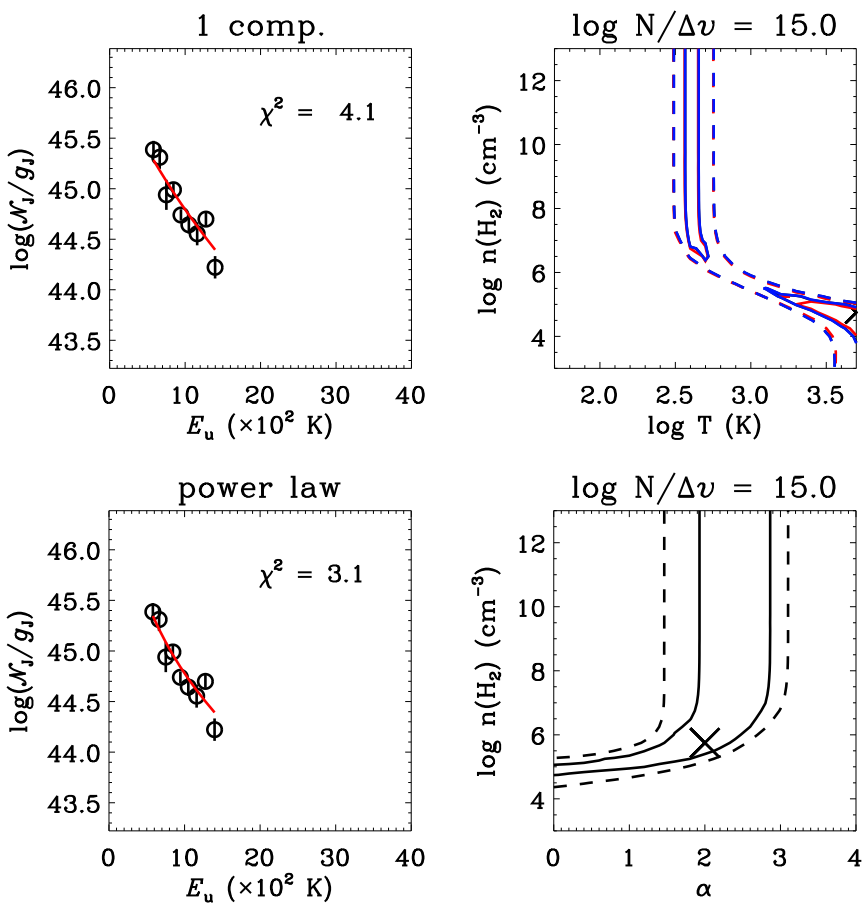

Figure 11. Same as Figure 9 but for L1527.

(The complete figure set (4 images) and a color version of this figure are available in the online journal.)

On the other hand, L1489 is best fitted with the sub-thermal solution for both the one-component model and the power-law model because its rotation diagram shows less curvature than TMC1 and TMR1. For L1527 and TMC1-A, the fits for the two models are not very different; as seen in the $\chi^{2}$ plot (Figure 11), both thermal and sub-thermal solutions are possible both in the one-component model and in the power-law model although the best-fit model has been selected to have the lowest $\chi^{2}$ for Table 8 . This unconstrained result is caused by the lack of lines at $E_{\mathrm{u}}$ above $1800 \mathrm{~K}$ in the two sources. L1551-IRS5 shows a similar result to L1527 and TMC1-A. Generally speaking, the single-temperature, non-LTE models tend to favor the highest kinetic temperatures in the grid, so they are not well constrained. The power-law models, on the other hand, result in a better fit, with a reasonably well-constrained power-law index, but the densities are often relatively unconstrained on the high side, suggesting that the excitation is close to thermal. 
Table 9

The Best-fit LVG Models of $\mathrm{H}_{2} \mathrm{O}$

\begin{tabular}{lccccc}
\hline \hline & & L1489 & TMR1 & L1527 & TMC1 \\
\hline & OPR & $1.5 \pm 0.5$ & $1.9 \pm 0.6$ & $2.4 \pm 2.8$ & $1.3 \pm 0.5$ \\
\hline \multirow{4}{*}{ comp. } & $T(\mathrm{~K})$ & $1.0(3)^{\mathrm{a}}$ & $5.0(3)$ & $2.0(3)$ & $1.6(3)$ \\
& $n\left(\mathrm{H}_{2}\right)\left(\mathrm{cm}^{-3}\right)$ & $1.8(7)$ & $1.0(5)$ & $3.2(4)$ & $3.2(5)$ \\
& $N\left(\mathrm{H}_{2} \mathrm{O}\right) / \Delta \mathrm{vm}^{-2} /\left(\mathrm{km} \mathrm{s}^{-1}\right)$ & $3.2(16)$ & $1.0(17)$ & $3.2(16)$ & $3.2(16)$ \\
& $\mathrm{Scale} \mathrm{factor}$ & $1.8(-2)$ & $2.1(-1)$ & $9.9(-1)$ & $8.2(-2)$ \\
$\chi^{2}$ & 6.4 & 2.4 & 4.0 & 1.6 \\
\hline \multirow{4}{*}{ Power law } & $n\left(\mathrm{H}_{2}\right)\left(\mathrm{cm}^{-3}\right)$ & $1.0(12)$ & $1.0(9)$ & $3.2(7)$ & $1.8(8)$ \\
& $p$ & 2.0 & 1.6 & 0.2 & 1.2 \\
& $N / \Delta v\left(\mathrm{H}_{2} \mathrm{O}\right) \mathrm{cm}^{-2} /\left(\mathrm{km} \mathrm{s}^{-1}\right)$ & $1.0(13)$ & $1.0(15)$ & $1.0(15)$ & $1.0(13)$ \\
& $\mathrm{Scale} \mathrm{factor}$ & $1.7(1)$ & $2.9(-1)$ & $7.4(-2)$ & $4.3(0)$ \\
$\chi^{2}$ & 8.6 & 7.2 & 4.9 & 1.1 \\
\hline
\end{tabular}

Notes.

a $a(b) \equiv a \times 10^{b}$.

${ }^{\mathrm{b}}$ Scale factor $=$ AREA $\left(\operatorname{arcsec}^{2}\right) \times$ FWHM $\left(\mathrm{km} \mathrm{s}^{-1}\right)$.
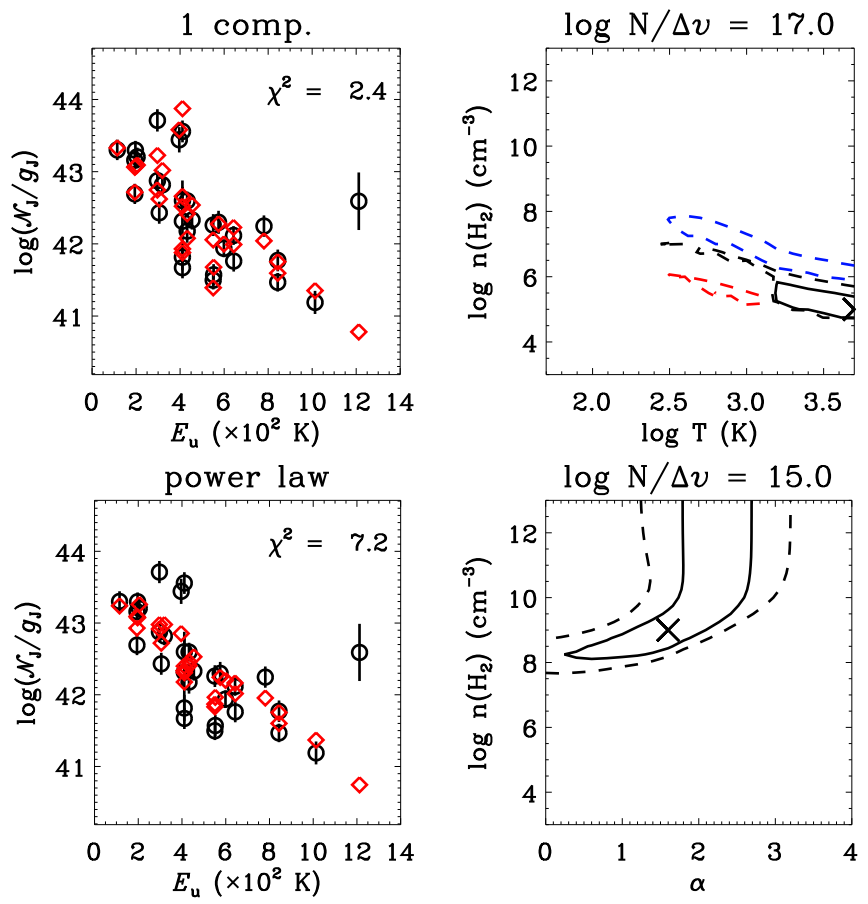

Figure 12. Best-fit RADEX models for $\mathrm{H}_{2} \mathrm{O}$ lines (top panels for the onecomponent model and bottom panels for the power-law temperature distribution model) in TMR1. The physical conditions for the models are summarized in Table 9. Diamonds without error bars indicate model points. Contours for $\chi^{2}$ are the same as in Figure 9.

(The complete figure set ( 8 images) and a color version of this figure are available in the online journal.)

\subsection{2. $\mathrm{H}_{2} \mathrm{O}$}

We also modeled the $\mathrm{H}_{2} \mathrm{O}$ fluxes with RADEX considering both one-component and power-law temperature distribution as was done for CO. The OPRs found in the rotational diagrams have been adopted. The $\chi^{2}$ and physical properties are similar for the best-fit models with different OPRs between 1 and 3 .

For the one-component model, we explored the same physical range as $\mathrm{CO}$. For the power-law model, we used the temperature range of $10 \mathrm{~K}$ to $5000 \mathrm{~K}$ as $\mathrm{CO}$, but the total $N\left(\mathrm{H}_{2} \mathrm{O}\right) / \Delta v$ ranges from $10^{13}$ to $10^{15} \mathrm{~cm}^{-2} \mathrm{~km}^{-1} \mathrm{~s}^{-1}$. The results for our best models are summarized in Table 9, and Figure 12 presents the best-models for TMR1, which are the richest in water lines among our sources.
The one-component model fits observations better than the power-law model for water, unlike CO. The power-law model is meaningful only when lines are optically thin because we do not have information on how the gas components with different temperatures distribute along the line of sight and the projected space. For the best-fit models, this condition is satisfied by $\mathrm{CO}$ lines, but half of water lines are optically thick in half of our sources. Therefore, the power-law temperature distribution in the one-dimensional physical structure cannot provide a good model for water lines. The gas temperatures of the best-fit onecomponent models for $\mathrm{H}_{2} \mathrm{O}$ are much greater than the rotational temperatures derived from its rotation diagrams, i.e., water is sub-thermally excited because of its high critical density. The densities $\left(10^{4} \sim 10^{7} \mathrm{~cm}^{-3}\right)$ of our best-fit models are much lower than the critical densities of many of water lines at the PACS range, which can reach up to $10^{11} \mathrm{~cm}^{-3}$ (Herczeg et al. 2012).

The best-fit models for $\mathrm{CO}$ (power-law model) and $\mathrm{H}_{2} \mathrm{O}$ (onecomponent model) indicate that $\mathrm{CO}$ and $\mathrm{H}_{2} \mathrm{O}$ are not excited by the same gas component; models that fit the $\mathrm{CO}$ do not fit the $\mathrm{H}_{2} \mathrm{O}$. CO seems to be excited by the warm gas mostly (at least for TMC1 and TMR1), but $\mathrm{H}_{2} \mathrm{O}$ is excited by the hot and dense gas. However, the one-dimensional power-law temperature model cannot deal with appropriately optically thick lines, and most water lines are optically thick.

\subsection{3. $\mathrm{OH}$}

It has been suggested that IR pumping could have an effect on the population of $\mathrm{OH}$ (Wampfler et al. 2013), so we included FIR radiation in our model. We considered FIR radiation by including blackbody radiation from the inner boundary of the envelope on top of the cosmic background radiation, which is implemented in RADEX. The temperature at the inner boundary of the envelope is assumed to be $250 \mathrm{~K}$. In order to calculate the dilution of the IR radiation from the central source to the given density, we assumed that the envelope has a power-law density structure with $n=n_{0} \times\left(r / r_{0}\right)^{-p}\left(r_{0}=\right.$ inner radius at $\mathrm{AU}$, $n_{0}=$ density at $\left.r_{0} \mathrm{~cm}^{-3}\right)$, and $r_{0}, n_{0}$, and $p$ were adopted from Kristensen et al. (2012). For more details of our inclusion of the central IR source, refer to Lee et al. (2013). We modeled the range of $50 \mathrm{~K} \leqslant T_{\mathrm{K}} \leqslant 250 \mathrm{~K}, 10^{3} \mathrm{~cm}^{-3} \leqslant n\left(\mathrm{H}_{2}\right) \leqslant$ (maximum density of the envelope model $)$, and $10^{10} \mathrm{~cm}^{-2} /\left(\mathrm{km} \mathrm{s}^{-1}\right) \leqslant$ $N(\mathrm{OH}) / \Delta v \leqslant 3.2 \times 10^{18} \mathrm{~cm}^{-2} /\left(\mathrm{km} \mathrm{s}^{-1}\right)$. 

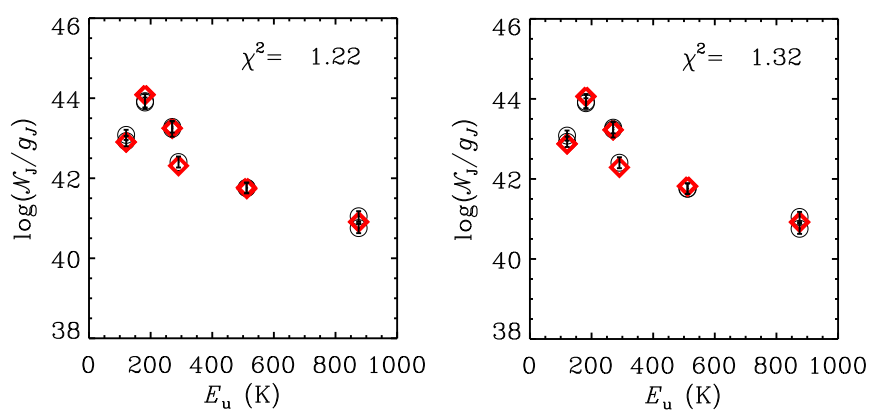

Figure 13. Best-fit RADEX models for OH lines in TMC1; left: the best-fit LVG model with the IR-pumping effect and right: the best-fit LVG model without the IR-pumping effect. The physical conditions for the models are summarized in Table 10. Symbols are the same as in Figure 12.

(The complete figure set ( 6 images) and a color version of this figure are available in the online journal.)

Table 10

The Best-fit LVG Models of OH

\begin{tabular}{|c|c|c|c|c|}
\hline & & L1489 & TMR1 & $\overline{\text { TMC1 }}$ \\
\hline \multirow{5}{*}{ Including FIR } & $T(\mathrm{~K})$ & 150 & 150 & 75 \\
\hline & $n\left(\mathrm{H}_{2}\right)\left(\mathrm{cm}^{-3}\right)$ & $2.5(8)^{\mathrm{a}}$ & $4.0(8)$ & $6.0(7)$ \\
\hline & $N(\mathrm{OH}) / \Delta \mathrm{v} \mathrm{cm}^{-2} /\left(\mathrm{km} \mathrm{s}^{-1}\right)$ & $1.0(15)$ & $1.8(15)$ & $1.8(17)$ \\
\hline & Scale factor ${ }^{\mathrm{b}}$ & $1.7(-1)$ & $2.1(-1)$ & $3.1(-1)$ \\
\hline & $\chi^{2}$ & 1.0 & 0.6 & 1.2 \\
\hline \multirow{5}{*}{ Not incl. FIR } & $T(\mathrm{~K})$ & 175 & 175 & 75 \\
\hline & $n\left(\mathrm{H}_{2}\right)\left(\mathrm{cm}^{-3}\right)$ & $2.2(8)$ & $3.7(8)$ & $6.0(7)$ \\
\hline & $N(\mathrm{OH}) / \Delta \mathrm{v} \mathrm{cm}^{-2} /\left(\mathrm{km} \mathrm{s}^{-1}\right)$ & $1.8(15)$ & $1.8(15)$ & $3.2(18)$ \\
\hline & Scale factor & $1.1(-1)$ & $1.8(-1)$ & $2.9(-1)$ \\
\hline & $\chi^{2}$ & 1.1 & 0.7 & 1.3 \\
\hline
\end{tabular}

Notes.

a $a(b) \equiv a \times 10^{b}$

${ }^{\mathrm{b}}$ Scale factor $=$ AREA $\left(\operatorname{arcsec}^{2}\right) \times$ FWHM $\left(\mathrm{km} \mathrm{s}^{-1}\right)$.

We considered fits with and without the IR pumping effect only from the central IR source. The $\mathrm{OH}$ fluxes of all the sources could be explained by either model; IR pumping effect is not necessary to reproduce the observed $\mathrm{OH}$ fluxes (see Figure 13 and Table 10). Lee et al. (2013) showed that the IR pumping is important only for the $\mathrm{OH}$ line with the highest energy level of $875 \mathrm{~K}($ at $55.9 \mu \mathrm{m})$, which was not detected toward these sources except for TMC1. For TMC1 where the highest energy level $\mathrm{OH}$ line has been detected, the consideration of IR pumping lowers the best-fit $\mathrm{OH}$ column density by a factor of 10 , compared to the model without the IR pumping effect. However, a caveat exists in the modeling of $\mathrm{OH}$ fluxes; the collision rates for $\mathrm{OH}$ are limited to $T_{\mathrm{K}}<250 \mathrm{~K}$ in the current molecular data. As in $\mathrm{CO}$ and $\mathrm{H}_{2} \mathrm{O}$, OH might be also excited by a much hotter gas. In addition, unlike Wampfler et al. (2013), our model did not consider the IR radiation from the dust component mixed with gas in situ. The lower energy level transitions are mostly optically thick, so the external IR photons do not strongly affect those lines.

We conclude that ad hoc models with a single temperature or with a temperature power law can fit some molecules but not others. Clearly a self-consistent physically motivated model including shocks and radiation is needed to make further progress.

\section{L1551-IRS5}

L1551-IRS5 stands out among our targets as the most luminous in both continuum and lines. This source shows very

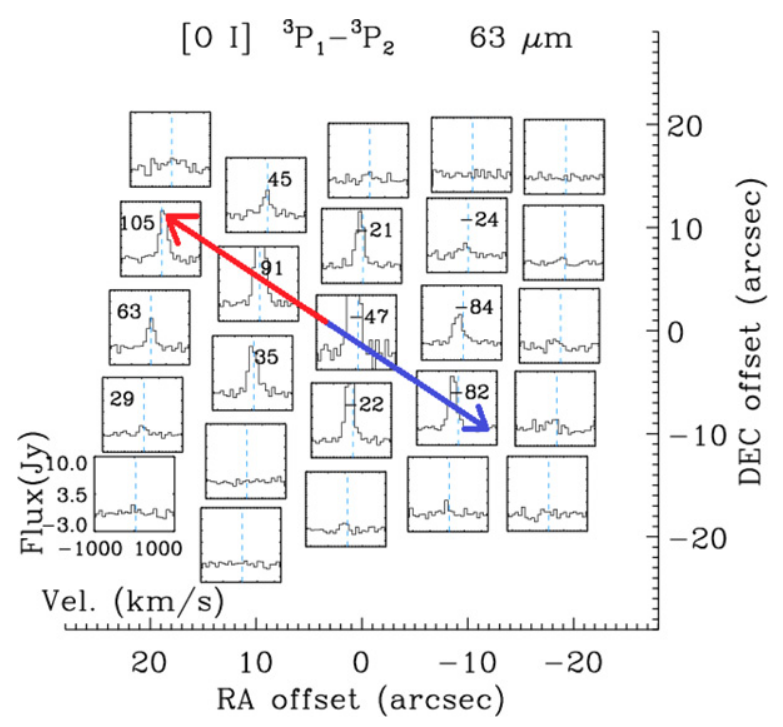

Figure 14. Central velocity shift of the [O I] $63.1 \mu \mathrm{m}$ line in L1551-IRS5. The central velocity is noted in the top of each spectrum. The marked red and blue arrows indicate the outflow direction for the redshifted and blueshifted components, respectively.

(A color version of this figure is available in the online journal.)

dry emission without any significant water line emission but with very strong $[\mathrm{O}$ I $]$ and $\left[\mathrm{C}_{\mathrm{II}}\right]$ emission. Similar characteristics (with mostly $\mathrm{CO}$ and $[\mathrm{OI}$ ] emission without much water emission) has been shown in more luminous protostars (Karska et al. 2014). The $\mathrm{OH}$ absorption line at $119 \mu \mathrm{m}$ has been detected toward L1551-IRS5. Wampfler et al. (2013) found the same absorption line feature toward several intermediate mass protostars (AFGL 490, Vela IRS 17, and Vela IRS 19), and one low-mass protostar, the Class 0 source, NGC 1333 IRS 2A (Wampfler et al. 2010). Karska et al. (2014) also found the $\mathrm{OH}$ absorption feature, even in transitions from higher energy levels, toward more luminous protostars.

L1551-IRS5 is a deeply embedded protostellar binary system (Rodriguez et al. 1998), and X-ray emission associated with $\mathrm{HH}$ 154 was discovered (Favata et al. 2002, 2006; Bally et al. 2003). The presence of X-ray sources in the vicinity of the protostar (Bonito et al. 2004; Favata et al. 2006; Schneider et al. 2011) suggests that high velocity jets $\left(v>500 \sim 1000 \mathrm{~km} \mathrm{~s}^{-1}\right)$ might have induced the X-ray emission, indicative of very fast shocks associated with L1551-IRS5.

Although the PACS spectra do not have sufficient spectral resolution to resolve the detailed kinematics in most sources, very fast outflows can make detectable velocity shifts. Figure 14 shows the [O I] $63 \mu \mathrm{m}$ line spectrum in each spaxel. The [O I] emission distributes along the known outflow direction. The number marked in the top of each box indicates the central velocity of the $[\mathrm{O} \mathrm{I}]$ line derived from the Gaussian profile fitting. The known source velocity $\left(v_{\text {LSR }}\right)$ is $+7.2 \mathrm{~km} \mathrm{~s}^{-1}$. Therefore, this map shows that the central velocity shifts up to $\pm 100 \mathrm{~km} \mathrm{~s}^{-1}$ approximately, with the redshifted emission in the NE and the blueshifted emission in the SW, which is consistent with the known outflow feature. Therefore, the velocity structure indicates that the [OI] emission is tracing the jet/wind in L1551-IRS5.

The $\mathrm{OH}$ absorption profile is shown in Figure 15 (left) as the optical depth versus wavelength. $\mathrm{OH}$ molecules in the dense envelope can produce this absorption feature due to the high FIR radiation field from the central source. We calculate the column 

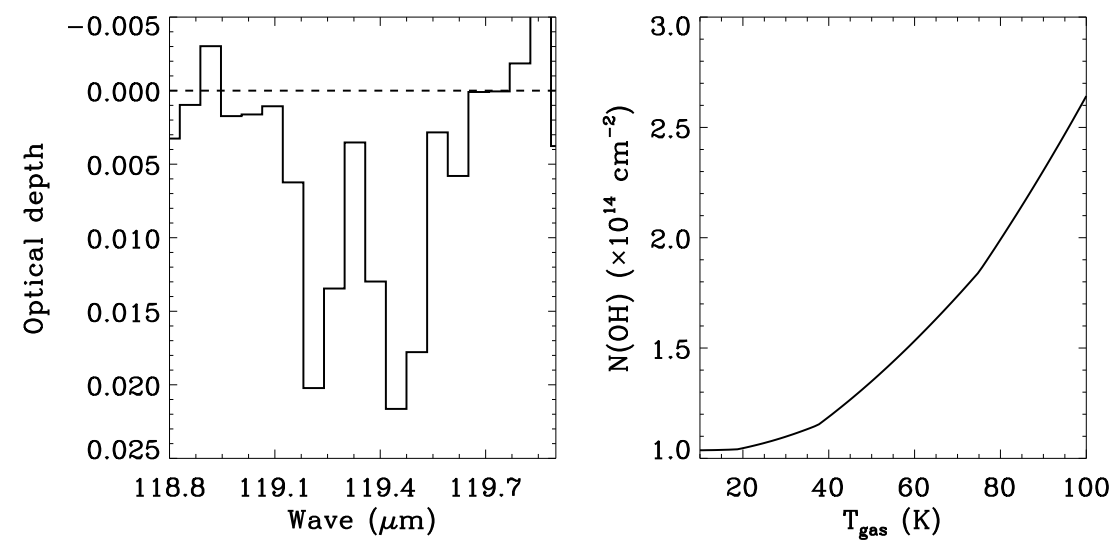

Figure 15. Left: the optical depth of the $\mathrm{OH} 119 \mu \mathrm{m}$ line in L1551-IRS5. Right: the $\mathrm{OH}$ column density, calculated from the optical depth profile, as a function of the kinetic temperature.

density of $\mathrm{OH}$ based on the LTE assumption using the equation below

$$
\int \tau_{\lambda} d \lambda=\frac{c}{v^{2}} \frac{c^{2}}{8 \pi v^{2}} A_{u l}\left[\frac{g_{u}}{g_{l}} x_{l}-x_{u}\right] N, x_{j}=\frac{g_{j}}{Q} e^{\left(-E_{j} / K T\right)},
$$

where $\tau$ is the observed optical depth, $N$ is the total column density, $Q(T)$ is the partition function, $T$ is the gas temperature, $c$ is the speed of light, and $g_{u}\left(g_{l}\right)$ and $E_{u}\left(E_{l}\right)$ is the statistical weight and the energy of upper (lower) level, respectively. $A_{u l}$ and $v$ are the Einstein coefficient and frequency, respectively, for this transition.

The column density needed to produce the absorption as a function of temperature is presented in Figure 15 (right). If the cold envelope $(T<20 \mathrm{~K})$ produces this absorption feature, then the lower limit of the $\mathrm{OH}$ column density in the cold envelope is about $1 \times 10^{14} \mathrm{~cm}^{-2}$.

\section{DISCUSSION}

\subsection{FIR Line Luminosities}

Our 6 sources show no significant trend in FIR line luminosity $\left(L_{\text {line }}\right)$ with either $L_{\mathrm{bol}}(r=0.53, p$ value $=0.26)$ or $T_{\mathrm{bol}}(r=$ $0.04, p$ value $=0.94)$. Karska et al. (2013) found a significant correlation $(r=0.71, p$ value $=0.0009)$ between $L_{\text {line }}$ and $L_{\text {bol }}$ at the $3 \sigma$ level with 18 sources. Therefore, the small sample in this work may obscure the actual correlation.

Our targets include one Class 0 and five Class I sources in the same environment. The Class 0 object, L1527, shows no special features compared to five other Class I sources. However, the most luminous source, L1551-IRS5 shows very different line luminosities compared to other less luminous sources; its FIR line luminosity is dominated by the [O I] lines. Thus we show correlation coefficients and fits without L1551-IRS5 in Figure 16 and Table 11.

The Pearson correlation coefficients at $3 \sigma$ (99.7\% confidence) and $2 \sigma$ (95.4\% confidence) levels for sample number of five are 0.98 and 0.89 , respectively. Therefore, if we consider only the five less luminous sources, molecular line luminosities correlate with each other at the $2 \sigma$ level, but no correlation with atomic line luminosities is seen (Figure 16, Table 11). However, the [O I] luminosity is correlated with the [C $\mathrm{II}]$ luminosity, indicative of the same heating mechanism. This correlation between [O I] and $[\mathrm{C} \mathrm{II}]$ luminosities is consistent with the similar spatial
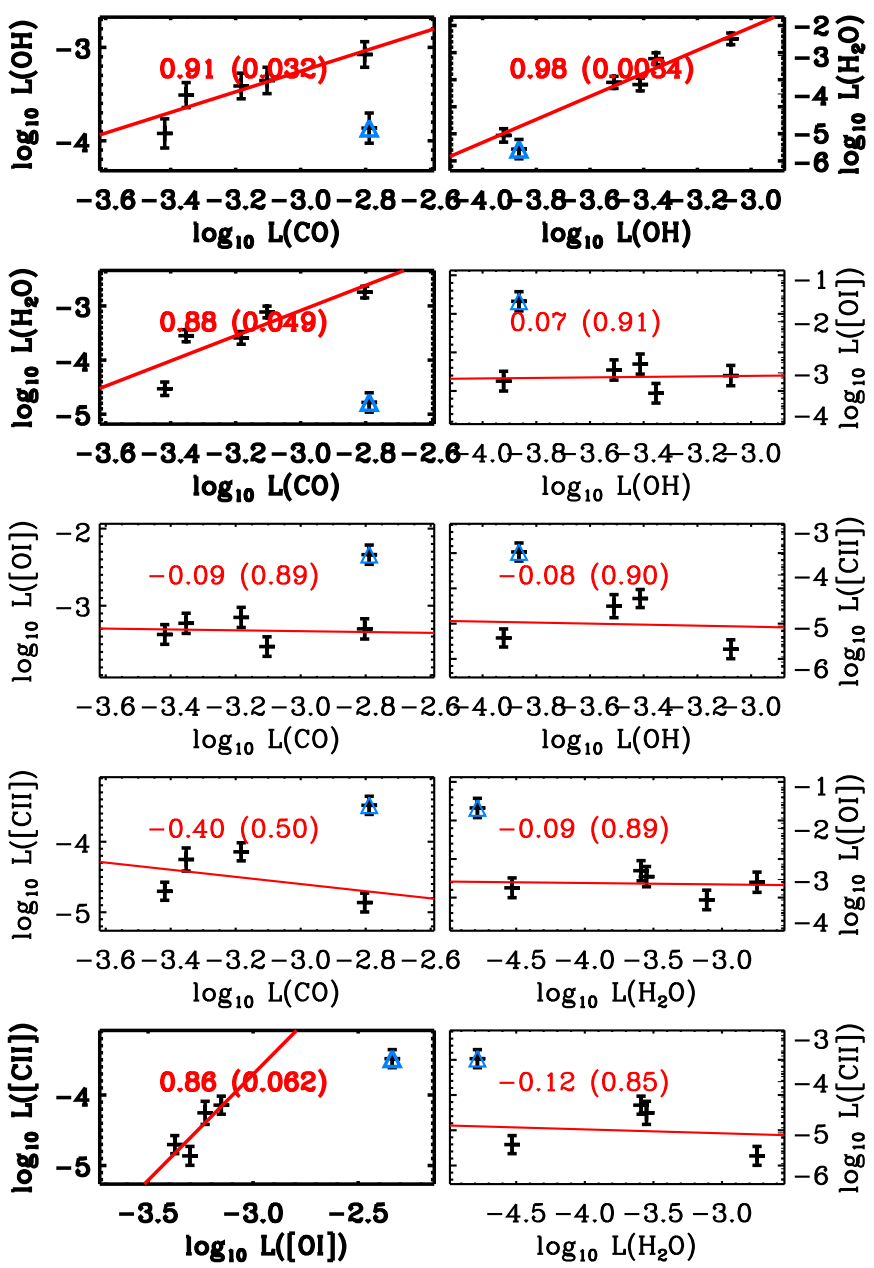

Figure 16. Correlations among line luminosities of different species. Luminosities are in the logarithmic scale. The blue triangle in each box indicates values for L1551-IRS5, which is not included in the correlation calculation. The boxes with thick lines represent species with some correlation. The red numbers in the upper left corner indicate the Pearson correlation coefficient with its $p$ value in parentheses.

(A color version of this figure is available in the online journal.)

distribution of the $[\mathrm{O} I]$ and $[\mathrm{C} I \mathrm{II}]$ line intensities shown in L1551-IRS5 and TMC1 (Figures 3 and 4). Therefore, the [C II] emission is possibly associated with the jet shock or the outflow cavity walls heated by the UV photons from the central source. 
Table 11

Pearson's Correlation Coefficients among Line Luminosites

\begin{tabular}{lccccc}
\hline \hline & $L_{\mathrm{CO}}$ & $L_{\mathrm{OH}}$ & $L_{\mathrm{H}_{2} \mathrm{O}}$ & $\left.L_{[\mathrm{O}}\right]$ & $L_{\left[\mathrm{C}_{\mathrm{II}}\right]}{ }^{\mathrm{a}}$ \\
\hline$L_{\mathrm{CO}}$ & $\ldots$ & $+0.91\left(0.032^{\mathrm{b}}\right)$ & $+0.88(0.049)$ & $-0.09(0.89)$ & $-0.40(0.50)$ \\
$L_{\mathrm{OH}}$ & & $\ldots$ & $+0.98(0.0034)$ & $+0.07(0.91)$ & $-0.08(0.90)$ \\
$L_{\mathrm{H}_{2} \mathrm{O}}$ & & & $\ldots$ & $-0.09(0.89)$ & $-0.12(0.85)$ \\
$L_{\mathrm{O} \text { I }}$ & & & & $\ldots$ & $+0.86(0.062)$ \\
$L_{\mathrm{C}}$ & & & & & $\ldots$ \\
\hline
\end{tabular}

Notes.

${ }^{\text {a }}$ L1489 was not detected in [C II].

${ }^{\mathrm{b}} p$ value.

\subsection{Origins of FIR Line Emission}

As shown Green et al. (2013) and Karska et al. (2013), the $\mathrm{CO}$ rotation diagrams for YSOs are commonly fitted by two components (warm and hot). Manoj et al. (2013) showed that the curvature in the $\mathrm{CO}$ rotation diagram could be explained either by a hot gas with low densities, which possibly exists inside the outflow cavity, or a gas with a power-law temperature distribution. Data on DIGIT sources, including L1448-MM (Lee et al. 2013) and GSS30-IRS1 (H. Je et al. in preparation), seem to prefer the latter solution.

Visser et al. (2012) combined the photodissociation region (PDR) and a $\mathrm{C}$ shock along the outflow cavity walls to fit the PACS CO line fluxes of several embedded protostars; they attributed the warm $\mathrm{CO}\left(T_{\text {rot }}=300 \mathrm{~K}\right)$ and the hot $\mathrm{CO}$ component of $T_{\text {rot }} \sim 1000 \mathrm{~K}$, respectively to the PDR and $\mathrm{C}$ shock. The $300 \mathrm{~K}$ component appears in the PACS CO rotation diagram for almost all embedded YSOs regardless of their luminosities, however, Manoj et al. (2013) and Karska et al. (2013) argued that the PDR has a minor contribution to the CO emission in the PACS range.

Surprisingly, according to our analysis, the relative contribution of $\mathrm{CO}$ to the total gas cooling is kept approximately constant. The relative contribution of $\mathrm{CO}$ to the total gas cooling in the PACS range is about $30 \%$ within errors in our six sources (see Figure 7) as well as in L1448-MM (Lee et al. 2013) and GSS30-IRS1 (H. Je et al. in preparation). Somehow, the relative contribution of $\mathrm{CO}$ to the total gas cooling is kept approximately constant.

We compared the line fluxes of our targets with the model fluxes for shocks calculated by Flower \& Pineau des Forêts (2010). The [O I] $63 / 145 \mu \mathrm{m}$ ratio of all sources is consistent with the $\mathrm{C}$ shock model (Figure 17). According to Kristensen et al. (2012), our sources have $n\left(\mathrm{H}_{2}\right) \sim 2 \times 10^{5} \mathrm{~cm}^{-3}$ at the radius of $1000 \mathrm{AU}$. Figure 18 shows the [O I] 63/145 $\mu \mathrm{m}$ ratio as a function of $L_{\mathrm{bol}}$; the ratio increases with $L_{\mathrm{bol}}$. In $\mathrm{C}$ shock models, the ratio increases with shock velocity, at least at the density of $2 \times 10^{5} \mathrm{~cm}^{-3}$ as seen in Figure 17 . Therefore, a higher $L_{\text {bol }}$ may result in a $\mathrm{C}$ shock with a higher velocity.

The actual intensity of [O I] lines can be calculated with an assumption of emitting area, which we adopt as the total size of spaxels where the [OI] emission is detected. The average [O I] $63 \mu \mathrm{m}$ intensity is $5.6 \times 10^{-5}, 2.5 \times 10^{-4}, 9.4 \times 10^{-5}$, $5.3 \times 10^{-5}, 1.1 \times 10^{-4}$, and $7.2 \times 10^{-5} \mathrm{erg} \mathrm{cm}^{-2} \mathrm{~s}^{-1} \mathrm{sr}^{-1}$ for L1489, L1551-IRS5, TMR1, TMC1-A, L1527, and TMC1, respectively. When compared with the shock models of Flower \& Pineau des Forêts (2010) (Figure 19), the average intensity is consistent more with $\mathrm{J}$ shock models $\left(15<v<25 \mathrm{~km} \mathrm{~s}^{-1}\right)$ for all sources. However, if a $\mathrm{C}$ shock in an irradiated environment (such as outflow cavity walls) is considered, the less luminous

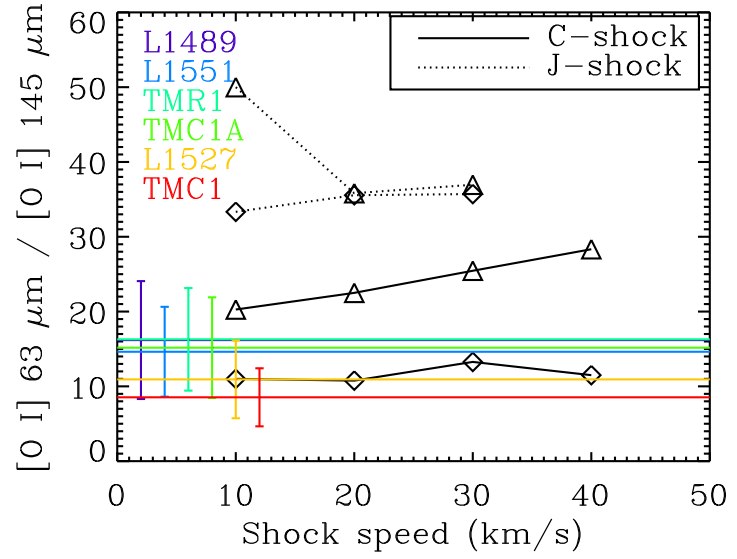

Figure 17. Comparison between the shock models by Flower \& Pineau des Forêts (2010) and the observations in the flux ratio between the [OI] 63 and $145 \mu \mathrm{m}$. The black lines and symbols represent the model results and the color lines show the observed values in our targets. Solid lines and dashed lines represent $\mathrm{C}$ and $\mathrm{J}$ shock models, respectively. Diamonds refer to $2 \times 10^{4} \mathrm{~cm}^{-3}$ and triangles to $2 \times 10^{5} \mathrm{~cm}^{-3}$ of hydrogen density.

(A color version of this figure is available in the online journal.)

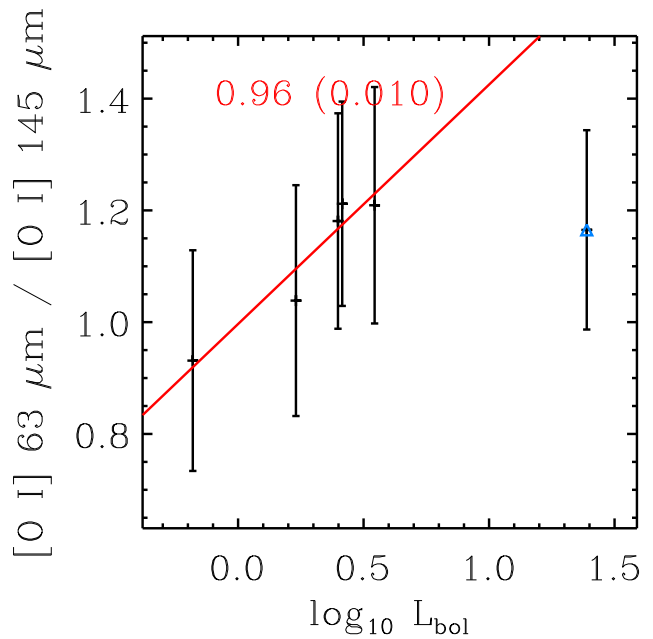

Figure 18. Correlation between flux ratio of [O I] $63 \mu \mathrm{m}$ and $145 \mu \mathrm{m}$ and $L_{\mathrm{bol}}$. The correlation was calculated by excluding L1551-IRS5, which is marked as a blue triangle. The red number at the upper left corner is the correlation coefficient with its $p$ value inside parentheses.

(A color version of this figure is available in the online journal.)

five sources might be fitted with a lower shock velocity. If a $\mathrm{J}$ shock was indeed important, the [O I] line ratio would indicate that the $63 \mu \mathrm{m}$ line was optically thick. However, the CO line fluxes are not well described by either $\mathrm{J}$ shock or $\mathrm{C}$ shock models as a whole.

Figures 20 and 21 present the luminosity ratio of $\mathrm{OH}$ and [O I], respectively, relative to that of $\mathrm{H}_{2} \mathrm{O}$. According to Lindberg et al. (2014), the high flux ratios of $\mathrm{OH} / \mathrm{H}_{2} \mathrm{O}$ and $[\mathrm{O} \mathrm{I}] / \mathrm{H}_{2} \mathrm{O}$ might be caused by the PDR process, which dissociates $\mathrm{H}_{2} \mathrm{O}$ to $\mathrm{OH}$ and $\mathrm{O}$, but their analysis was based on one specific line of each species. Although not very distinct in $\mathrm{OH} / \mathrm{H}_{2} \mathrm{O}$ luminosity ratio (Figure 20), our sources are readily divided into three groups in the $\left[\mathrm{O}_{\mathrm{I}}\right] / \mathrm{H}_{2} \mathrm{O}$ luminosity ratio (Figure 21 ); $L_{[\mathrm{OI}} /$ $L_{\mathrm{H}_{2} \mathrm{O}}<1$ in L1489 and TMR1, between 1 and a few $10 \mathrm{~s}$ (more conservatively, 100) for TMC1, L1527, and TMC1-A, and above 100 for L1551-IRS5. The group with $L_{[\mathrm{O} \text { I] }} / L_{\mathrm{H}_{2} \mathrm{O}}$ ratios greater than 1 is likely affected by photodissociation of water. A portion of $\mathrm{CO}$ must be dissociated in the same gas 

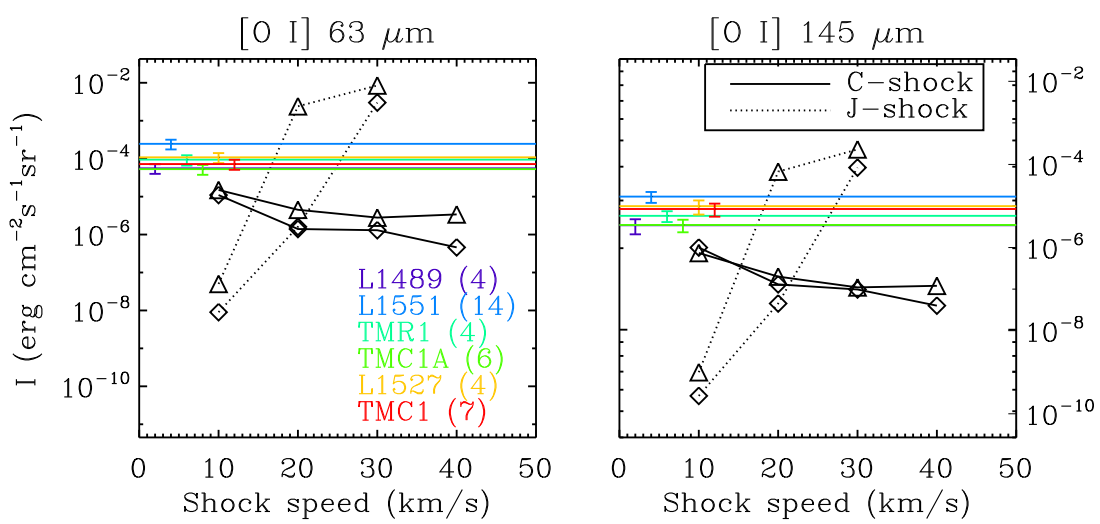

Figure 19. Average intensity of [O I] lines over emitting area for each source. The numbers in parentheses are the number of spaxels where the [O I] $63 \mu \mathrm{m}$ line was detected. Symbols and colors are the same as in Figure 17.

(A color version of this figure is available in the online journal.)
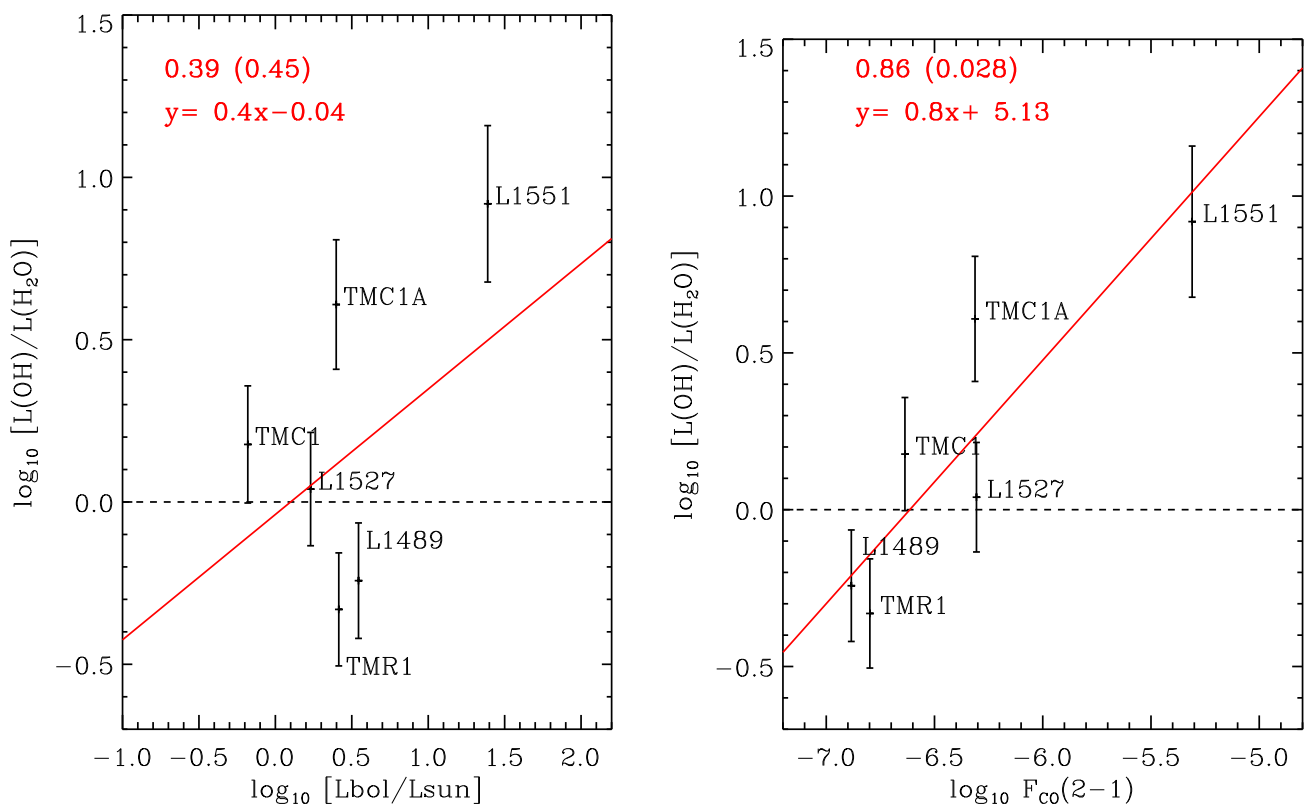

Figure 20. Luminosity ratios of $\mathrm{OH}$ relative to that of $\mathrm{H}_{2} \mathrm{O}$ with respect to $L_{\mathrm{bol}}$ and $F_{\mathrm{CO}}(2-1)$. The red numbers in the upper left corner indicate the correlation coefficients with their $p$ values inside parentheses. The equations given inside boxes present the fitting result of red lines. $F_{\mathrm{CO}}(2-1)$ are adopted from Kang et al. (in preparation).

(A color version of this figure is available in the online journal.)

component since the spatial distribution of the $[\mathrm{C} \mathrm{II}]$ emission is consistent with that of [OI] (at least for L1551-IRS5 and TMC1), and their luminosities have a correlation.

However, the origin of the high-energy photons does not need to be the central or external (proto-)stars. For instance, L1551-IRS5 is known to have shock-induced X-ray emission (Favata et al. 2002, 2006). Therefore, a dissociative J shock can also increase those $\mathrm{OH} / \mathrm{H}_{2}$ and $[\mathrm{O} I] / \mathrm{H}_{2} \mathrm{O}$ luminosity ratios. However, the low $L_{\mathrm{bol}}$ of TMC1, for example, indicates a lowmass accretion rate, and thus a very energetic shock capable of dissociating $\mathrm{H}_{2} \mathrm{O}$, is not expected. Indeed, as seen in Figure 20 (right) and Figure 21 (right), the outflow momentum flux $\left(F_{\mathrm{CO}}\right)$ of L1551-IRS5 is much larger than the other sources. Therefore, the two groups with the ratios lower than 100 may be explained by $\mathrm{C}$ shocks. The middle group with the ratios greater than 1 and smaller than $\sim 100$ may have been influenced by UV photons produced by the accretion to the central protostar in addition to $\mathrm{C}$ shocks, i.e., irradiated $\mathrm{C}$ shocks may be important for the middle group. Therefore, the ratio of $L_{\left[\mathrm{O}_{\mathrm{I}}\right]} / L_{\mathrm{H}_{2} \mathrm{O}}$ may characterize the heating mechanisms in the YSO envelopes; $\sim 100$ for the division between $\mathrm{J}$ and $\mathrm{C}$ shock and 1 for the division between an irradiated $\mathrm{C}$ shock and $\mathrm{C}$ shock without irradiation.

One more important point from Figures 20 and 21 is that these ratios of $L_{\left[\mathrm{O}_{\mathrm{I}}\right]} / L_{\mathrm{H}_{2} \mathrm{O}}$ and $L_{\mathrm{OH}} / L_{\mathrm{H}_{2} \mathrm{O}}$ are better correlated with $F_{\mathrm{CO}}(r=0.94$ with $p$ value $=0.0052$ and $r=0.86$ with $p$ value $=$ $0.028)$ than $L_{\text {bol }}(r=0.51$ with $p$ value $=0.30$ and $r=0.39$ with $p$ value $=0.45) . F_{\mathrm{CO}}$ traces the integrated activity over the entire lifetime of the YSO, but $L_{\mathrm{bol}}$ is associated more with the current accretion process. Therefore, it is interesting to find a tighter correlation of $F_{\mathrm{CO}}$ with the currently shocked gas probed by the FIR [O I], OH, and $\mathrm{H}_{2} \mathrm{O}$ observations. According to this result, the FIR line luminosity ratio between [O I] (or $\mathrm{OH}$ ) and $\mathrm{H}_{2} \mathrm{O}$ should reflect the time averaged outflow properties.

According to the PDR model by Lee et al. (2014), CO can have an abundance greater than $10^{-5}$ at $n\left(\mathrm{H}_{2}\right)$ of $10^{6}$ to $10^{8} \mathrm{~cm}^{-3}$ if the gas temperature is greater than $300 \mathrm{~K}$. These conditions may arise at the cavity wall surface $\left(A_{\mathrm{V}}<0.5 \mathrm{mag}\right)$ when 

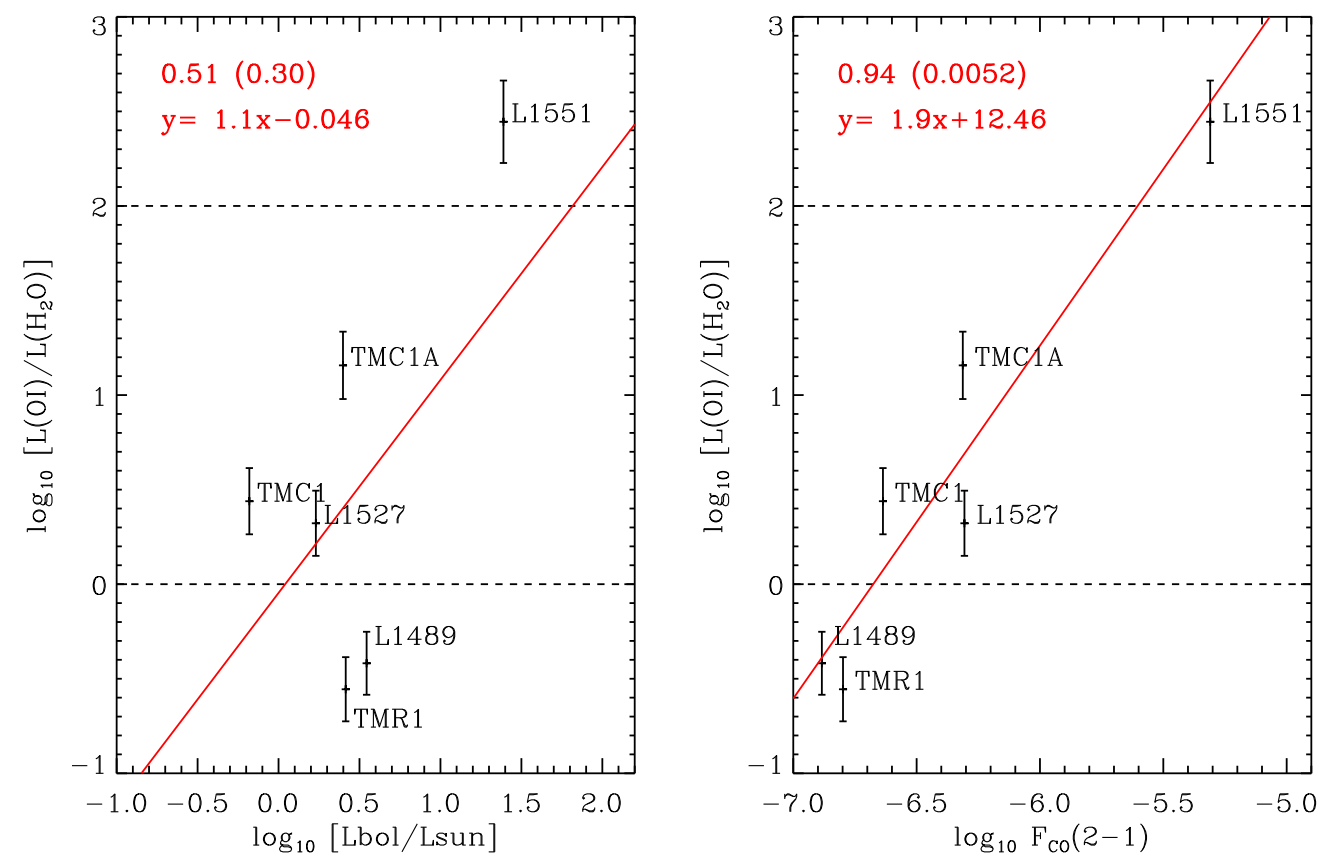

Figure 21. Luminosity ratios of [O I] relative to that of $\mathrm{H}_{2} \mathrm{O}$ with respect to $L_{\mathrm{bol}}$ and $F_{\mathrm{CO}}(2-1)$. The numbers are the same as in Figure 20 .

(A color version of this figure is available in the online journal.)

the ratio of $\mathrm{UV}$ flux $\left(G_{0}\right.$; in the unit of the average ISRF of $1.6 \times 10^{-3}$ erg cm $\mathrm{cm}^{-2} \mathrm{~s}^{-1}$ ) to density $(n), G_{0} / n$ is $\sim 10^{-3} \mathrm{~cm}^{3}$. Certainly, the gas in the cavity wall has been shocked and must have high velocities as observed with HIFI (Kristensen et al. 2012). Therefore, the kinematical, physical, and chemical conditions along the outflow cavity wall cannot be explained solely either by PDR or shock. Further investigation with more sophisticated models for irradiated shocks is necessary.

\section{SUMMARY}

The DIGIT observations of six Taurus sources with the PACS SED range mode have been analyzed.

1. The FIR continuum luminosity $\left(L_{\text {cont }}\right)$ has a very strong correlation with $L_{\text {bol }}$, but the richness of line emission $\left(L_{\text {line }}\right)$ is not related to $L_{\mathrm{bol}}$. The main difference between sources is the amount of water emission, which is relatively low in the most luminous sources. L1489, TMR1, and TMC1, which are the faintest sources among our targets, are rich in water lines while L1551-IRS5 and L1527, which are known as strong outflow sources, do not show much water emission.

2. Molecular emission is compact in these sources, but atomic emission such as [O I] and [C II] tends to be extended along the known outflow directions.

3. The relative line luminosity of each species varies by source, although CO consistently contributes $\sim 30 \%$ to the total FIR line cooling.

4. Similar to previous studies, the rotation diagram of $\mathrm{CO}$ shows is well characterized by two temperature components of $\sim 350 \mathrm{~K}$ and $\geqslant 700 \mathrm{~K}$. The rotation diagrams of $\mathrm{OH}$ and $\mathrm{H}_{2} \mathrm{O}$ can be fitted by a single component between $100 \mathrm{~K}$ and $200 \mathrm{~K}$.

5. The non-LTE LVG models of CO indicate that models with temperature gradients are the most plausible. In order to fit our $\mathrm{H}_{2} \mathrm{O}$ fluxes, hot gas with kinetic temperatures above $1000 \mathrm{~K}$, which is much higher than the excitation temperatures derived from the rotation diagrams, is required. The
$\mathrm{OH}$ fluxes can be fitted by models either with or without the IR-pumping effect from the central source.

6. Among our sources, L1551-IRS5 seems to have different physical and chemical conditions. Approximately $70 \%$ of its FIR line luminosity appears in two [O I] lines, with almost no accompanying water emission. This strongly suggests that the very fast $\mathbf{J}$ shock is working in L1551IRS5 to dissociate water to atomic oxygen. In addition, the [O I] emission is distributed along the outflow direction with the consistent shift of the central velocity, probably induced by jet-shocks. In L1551-IRS5, the OH $119 \mu \mathrm{m}$ line shows absorption, which might be produced by the $\mathrm{OH}$ column density of $\geqslant 1 \times 10^{14} \mathrm{~cm}^{-2}$.

7. The FIR line luminosities of each molecular species correlate with each other among the less luminous five sources at the confidence greater than $95 \%$. The [O I] and [C II] line luminosities correlated with each other ( $94 \%$ confidence) but not with other molecular luminosities.

8. It is difficult to designate one heating mechanism to explain all line emission in the PACS range. It is unrealistic to assume that only the PDR or shock alone heats the gas in the embedded sources. Rather the combination of the two processes, i.e., irradiated shocks must produce the FIR line emission for most sources. However, the shock properties, and thus, the resulting chemistry must be very different in L1551-IRS5 from the other five sources; a dissociative $\mathrm{J}$ shock likely controls the energetics and its chemistry in L1551-IRS5.

9. The line luminosity ratio, $L_{\left[\mathrm{O}_{\mathrm{I}}\right]} / L_{\mathrm{H}_{2} \mathrm{O}}$ can be used to characterize the heating mechanisms in the YSO envelopes; $\sim 100$ for the division between $\mathrm{J}$ and $\mathrm{C}$ shock and 1 for the division between irradiated $\mathrm{C}$ shock and normal $\mathrm{C}$ shock.

We thank A. Karska and the anonymous referee, whose comments led to improvements in the paper. J.-E. L. is very grateful to the department of Astronomy, University of Texas at Austin for the hospitality provided to her from 2013 August to 
2014 July. J.-E. L. was supported by the LG Yonam Foundation Oversea Research Professor Program in 2013. Support for this work, part of the Herschel Open Time Key Project Program, was provided by NASA through an award issued by the Jet Propulsion Laboratory, California Institute of Technology. This research was supported by the Basic Science Research Program through the National Research Foundation of Korea (NRF) funded by the Ministry of Education of the Korean government (grant No. NRF-2012R1A1A2044689) and the 2013 Sabbatical Leave Program of Kyung Hee Unviersity (KHU-20131724, 20131727).

\section{REFERENCES}

Bally, J., Feigelson, E., \& Reipurth, B. 2003, ApJ, 584, 843

Bonito, R., Orlando, S., Peres, G., et al. 2004, A\&A, 424, 1

Bontemps, S., Andre, P., Terebey, S., et al. 1996, A\&A, 311, 858

Curtis, E. I., Richer, J. S., Swift, J. J., \& Williams, J. P. 2010, MNRAS, 408, 1516

de Graauw, Th., Helmich, F. P., Phillips, T. G., et al. 2010, A\&A, 518, 6

Dionatos, O., Jørgensen, J., Green, J., et al. 2013, A\&A, 558, 88

Dunham, M. M., Crapsi, A., Evans, N. J., et al. 2008, ApJS, 179, 249

Favata, F., Bonito, R., Micela, G., et al. 2006, A\&A, 450, 17

Favata, F., Fridlund, C. V. M., Micela, G., et al. 2002, A\&A, 386, 204

Flower, D. R., \& Pineau des Forêts, G. 2010, MNRAS, 406, 1745

Goicoechea, J. R., Cernicharo, J., Karska, A., et al. 2012, A\&A, 548, 77

Green, J., Evan, N., Jørgensen, J., et al. 2013, ApJ, 770, 123

Herczeg, G. J., Karska, A., Bruderer, S., et al. 2012, A\&A, 540, 84

Hogerheijde, M. R., van Dishoeck, E. F., Blake, G. A., et al. 1998, ApJ, 502,315
Karska, A., Herczeg, G. J., van Dishoeck, E. F., et al. 2013, A\&A, 552, 141

Karska, A., Herpin, F., Bruderer, S., et al. 2014, A\&A, 562, 45

Kaufman, M. J., Wolfire, M. G., Hollenbach, D. J., \& Luhman, M. L. 1999, ApJ, 527,795

Kristensen, L. E., van Dishoeck, E. F., Bergin, E. A., et al. 2012, A\&A, 542, A8

Kristensen, L. E., van Dishoeck, E. F., Tafalla, M., et al. 2011, A\&A, $531, \mathrm{~L} 1$

Lee, S., Lee, J.-E., Bergin, E. A., \& Park, Y.-S. 2014, ApJS, 213, 33

Lee, J., Lee, J.-E., Lee, S., et al. 2013, ApJS, 209, 4

Lindberg, J. E., Jørgensen, J., Green, J., et al. 2014, A\&A, 565, A29

Mamon, G. A., Glassgold, A. E., \& Huggins, P. J. 1988, ApJ, 328, 797

Manoj, P., Watson, D. M., Neufeld, D. A., et al. 2013, ApJ, 763, 83

Mumma, M. J., Weaver, H. A., \& Larson, H. P. 1987, A\&A, 187, 419

Narayanan, G., Snell, R., \& Bemis, A. 2012, MNRAS, 425, 2641

Neufeld, D. A. 2012, ApJ, 749, 125

Neufeld, D. A., Gusdorf, A., Güsten, R., et al. 2014, ApJ, 781, 102

Nisini, B., Giannini, T., \& Lorenzetti, D. 2002, ApJ, 574, 246

Pilbratt, G., Riedinger, J. R., Passvogel, T., et al. 2010, A\&A, 518, 1

Poglitsch, A., Waelkens, C., Geis, N., et al. 2010, A\&A, 518, 2

Rodriguez, L. F., D’Alessio, P., Wilner, D. J., et al. 1998, Natur, 395, 355

Schneider, P. C., Gunther, H. M., Schmitt, J. H. M. M., et al. 2011, A\&A, 530,123

Schoier, F. L., van der Tak, F. F. S., van Dishoeck, E. F., et al. 2005, A\&A, 432, 369

Shu, F., Adams, F., \& Lizano, S. 1987, ARA\&A, 25, 23

Snell, R. L., Hollenbach, D., Howe, J. E., et al. 2005, ApJ, 620, 758

Tobin, J. J., Hartmann, L., Calvet, N., \& DÁlessio, P. 2008, ApJ, 679, 1364

van der Tak, F. F. S., Black, J. H., Schoier, F. L., et al. 2007, A\&A, 468, 627

Visser, R., Kristensen, L. E., Bruderer, S., et al. 2012, A\&A, 537, 55

Wampfler, S. F., Bruderer, S., Karska, A., et al. 2013, A\&A, 552, 56

Wampfler, S. F., Herczeg, G. J., Bruderer, S., et al. 2010, A\&A, 521, 36

Wu, P.-F., Takakuwa, S., \& Lim, J. 2009, ApJ, 698, 184 